\title{
Radiative decays of charged leptons as constraints of unitarity polygons for active-sterile neutrino mixing and $\mathrm{CP}$ violation
}

\author{
Zhi-zhong Xing ${ }^{1,2}$, Di Zhang ${ }^{1, a}$ \\ ${ }^{1}$ Institute of High Energy Physics and School of Physical Sciences, University of Chinese Academy of Sciences, Beijing 100049, China \\ ${ }^{2}$ Center of High Energy Physics, Peking University, Beijing 100871, China
}

\begin{abstract}
We calculate the rates of radiative $\beta^{-} \rightarrow \alpha^{-}+\gamma$ decays for $(\alpha, \beta)=(e, \mu),(e, \tau)$ and $(\mu, \tau)$ by taking the unitary gauge in the $(3+n)$ active-sterile neutrino mixing scheme, and make it clear that constraints on the unitarity of the $3 \times 3$ Pontecorvo-Maki-Nakagawa-Sakata (PMNS) matrix $U$ extracted from $\beta^{-} \rightarrow \alpha^{-}+\gamma$ decays in the minimal unitarity violation scheme differ from those obtained in the canonical seesaw mechanism with $n$ heavy Majorana neutrinos by a factor $5 / 3$. In such a natural seesaw case we show that the rates of $\beta^{-} \rightarrow \alpha^{-}+\gamma$ can be used to cleanly and strongly constrain the effective apex of a unitarity polygon, and compare its geometry with the geometry of its three sub-triangles formed by two vectors $U_{\alpha i} U_{\beta i}^{*}$ and $U_{\alpha j} U_{\beta j}^{*}$ (for $i \neq j$ ) in the complex plane. We find that the areas of such sub-triangles can be described in terms of the Jarlskog-like invariants of $\mathrm{CP}$ violation $\mathcal{J}_{\alpha \beta}^{i j}$, and their small differences signify slight unitarity violation of the PMNS matrix $U$.
\end{abstract}

\section{Introduction}

The discoveries of solar, atmospheric, reactor and accelerator neutrino oscillations [1] have changed some of our previous ideas about the standard model (SM) of particle physics, especially about its lepton flavor sector. Now we are firmly convinced that neutrinos have mysteriously tiny masses and lepton flavors are significantly mixed. Behind these two important observations must be some kind of new physics beyond the SM [2], which is responsible for the origin of neutrino masses and may have far-reaching implications for particle physics and cosmology.

In weak charged-current interactions it is the $3 \times 3$ Pontecorvo-Maki-Nakagawa-Sakata (PMNS) matrix $U$ that describes the effects of lepton flavor mixing and $\mathrm{CP}$ violation [3-7]. The smallest element of $U$ is found to be $\left|U_{e 3}\right| \simeq 0.15$

\footnotetext{
a e-mail: zhangdi@ihep.ac.cn (corresponding author)
}

[8], much larger than $\left|V_{u b}\right| \simeq 3.6 \times 10^{-3}$ - the smallest element of the $3 \times 3$ Cabibbo-Kobayashi-Maskawa (CKM) quark flavor mixing matrix $V[9,10]$. Moreover, the PMNS matrix $U$ is very likely to accommodate significant $\mathrm{CP}$ violation in the lepton sector, as indicated by the latest $\mathrm{T} 2 \mathrm{~K}$ measurement at almost the $3 \sigma$ confidence level [11]. Given the unitarity of $U$, there are three Dirac-type unitarity triangles defined by the orthogonality conditions $[12]^{1}$

$U_{\alpha 1} U_{\beta 1}^{*}+U_{\alpha 2} U_{\beta 2}^{*}+U_{\alpha 3} U_{\beta 3}^{*}=0$

in the complex plane, where $(\alpha, \beta)=(e, \mu),(\mu, \tau)$ or $(\tau, e)$. The geometry of each triangle depends only upon the Dirac phase $\delta$ of $U$ which gives rise to leptonic $\mathrm{CP}$ violation in those "appearance"-type long-baseline neutrino oscillations as recently constrained by the T2K experiment [11]. So far quite a lot of attention has been paid to leptonic unitarity triangles in vacuum or in matter [14-21], partly because such a geometric language has proved to be very successful in the quark sector to intuitively describe the most salient features of flavor mixing and $\mathrm{CP}$ violation.

But unlike the CKM matrix $V$, whose unitarity is guaranteed by the SM itself, whether the PMNS matrix $U$ is exactly unitary or not depends on the origin of neutrino masses. From a theoretical point of view, the most natural and popular mechanism of generating finite but tiny neutrino masses is the canonical seesaw mechanism [22-26] an extension of the SM by adding $n$ heavy (right-handed) neutrino fields and allowing lepton number violation. Such $\mathrm{SU}(2)_{\mathrm{L}}$-singlet neutrino fields are coupled with the leptonic $\mathrm{SU}(2)_{\mathrm{L}}$ doublet and the Higss doublet as an SM-like

\footnotetext{
1 There are also three Majorana-type triangles defined by the orthogonality relations $U_{e i} U_{e j}^{*}+U_{\mu i} U_{\mu j}^{*}+U_{\tau i} U_{\tau j}^{*}=0$ in the complex plane $[12,13]$, where $(i, j)=(1,2),(2,3)$ or $(3,1)$. The configuration of each triangle is sensitive to the relative phases of three Majorana neutrino mass eigenstates $v_{i}$ (for $i=1,2,3$ ) [16], but it is irrelevant to the radiative decays of charged leptons and hence will not be discussed in this paper.
} 
Yukawa interaction term, and they may also form a gaugeinvariant but lepton-number-violating Majorana mass term with their own charge-conjugated counterparts. After spontaneous gauge symmetry breaking, the resultant neutrino mass matrix is a $(3+n) \times(3+n)$ symmetric matrix whose eigenvectors correspond to three light Majorana neutrino fields $v_{i}$ (for $i=1,2,3$ ) and $n$ heavy Majorana neutrino fields $N_{j}$ (for $j=1,2, \ldots, n$ ). In this case the mixing between light (active) and heavy (sterile) neutrino flavors is described by a $3 \times n$ matrix $R[27,28]$, and it is correlated with the $3 \times 3$ PMNS matrix $U$ via $U U^{\dagger}+R R^{\dagger}=I$. Since both $U$ and $R$ are the sub-matrices of a $(3+n) \times(3+n)$ unitary matrix $\mathcal{U}$ used to diagonalize the overall $(3+n) \times(3+n)$ neutrino mass matrix, neither of them is exactly unitary. The standard weak charged-current interactions of three charged leptons and $(3+n)$ neutrinos turn out to be $[27,28]$

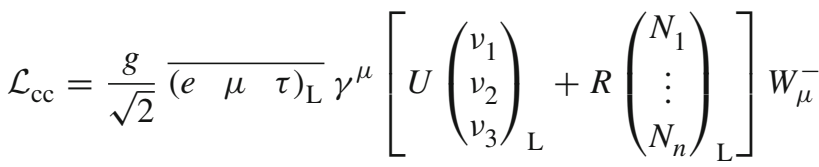

$$
\begin{aligned}
& \text { +h.c., }
\end{aligned}
$$

where both the charged leptons and neutrinos are in their mass eigenstates, and the correlation between $R$ and $U$ has been given above (i.e., $U U^{\dagger}=I-R R^{\dagger}$ ). As a straightforward consequence, the three Dirac-type unitarity triangles defined in Eq. (1) are now replaced with three unitarity polygons defined by the orthogonality relations

$U_{\alpha 1} U_{\beta 1}^{*}+U_{\alpha 2} U_{\beta 2}^{*}+U_{\alpha 3} U_{\beta 3}^{*}=-\sum_{i=1}^{n} R_{\alpha i} R_{\beta i}^{*}$

in the complex plane, where $(\alpha, \beta)=(e, \mu),(\mu, \tau)$ or $(\tau, e)$. In other words, the unitarity of $U$ is violated due to $R \neq 0$. Since the strength of active-sterile flavor mixing is expected to be very small in a natural seesaw model, the departure of a unitarity polygon from the corresponding unitarity triangle should also be very small and can serve as a clear signal of new physics if it is finally measured at low energies. Figure 1 schematically illustrates an effective Dirac-type unitarity triangle and four typical topologies of its apex in the complex plane, where pattern (a) corresponds to exact unitarity in the standard case and pattern (b1), (b2) or (b3) stands for slight unitarity violation in the presence of active-sterile neutrino mixing. Then the question becomes which weak-interaction process at low energies is appropriate for constraining a given unitarity polygon and probing its deviation from the corresponding unitarity triangle.

In the present paper we are going to show that the radiative decays of charged leptons, denoted as $\beta^{-} \rightarrow \alpha^{-}+\gamma$ for $(\alpha, \beta)=(e, \mu),(e, \tau)$ or $(\mu, \tau)$, can be used to impose a straightforward and strong constraint on the effective apex of a given unitarity polygon in the canonical seesaw mechanism with the heavy Majorana neutrino masses $M_{i}$ (for $i=1,2, \ldots, n)$ being far above the charged vector boson mass $M_{W}$. It is worth pointing out that our work is different from those previous studies (see Ref. [29] for a recent review with extensive references) in the following three aspects.

- We calculate the rates of lepton-flavor-violating $\beta^{-} \rightarrow$ $\alpha^{-}+\gamma$ decays mediated by both three active neutrinos and $n$ sterile neutrinos via Eq. (2) in the unitary gauge, where the sterile neutrinos can in general be either heavy or light (i.e., one is unnecessarily subject to the canonical seesaw mechanism). Our result is consistent with those obtained in Refs. [30,31]. ${ }^{2}$ Switching off the sterile neutrinos, we may immediately reproduce the pioneering results achieved in 1977 [22,32-37]. We make it clear that constraints on the unitarity of $U$ extracted from $\beta^{-} \rightarrow \alpha^{-}+\gamma$ decays in the so-called minimal unitarity violation scheme (see, e.g., Refs. [38-40]) are different from those obtained in the canonical seesaw mechanism with $n$ heavy Majorana neutrinos by a factor $5 / 3$, simply because there is a constant which dominates the loop function in the former case but it is cancelled out in the latter case due to the unitarity condition $U U^{\dagger}+R R^{\dagger}=I$.

- We illustrate how the loop function $G_{\gamma}\left(x_{i}\right)$ evolves with $x_{i} \equiv \lambda_{i}^{2} / M_{W}^{2}$, where $\lambda_{i}$ represents an arbitrary neutrino mass (for $i=1,2, \ldots, n+3$ ), and identify the two asymptotic convergence limits $G_{\gamma}\left(x_{i}\right) \rightarrow-1 / 3$ for $x_{i} \gg 1$ and $G_{\gamma}\left(x_{i}\right) \rightarrow-5 / 6$ for $x_{i} \ll 1$. In the former case we demonstrate that the rates of $\beta^{-} \rightarrow \alpha^{-}+\gamma$ can be used to cleanly constrain the effective apex of a unitarity polygon shown by Fig. 1 in the canonical seesaw mechanism, and in the latter case we propose to define three new effective neutrino masses which are directly sensitive to charged lepton flavor violation. Some numerical results are also obtained in these two cases.

- We explore the geometry of a given unitarity polygon as compared with the geometry of its three sub-triangles $\triangle_{\alpha \beta}^{i j}$ formed by two vectors $U_{\alpha i} U_{\beta i}^{*}$ and $U_{\alpha j} U_{\beta j}^{*}$ (for $i \neq$ $j)$ in the complex plane. The areas of such sub-triangles can be described in terms of the Jarlskog-like invariants of CP violation [41]

$$
\mathcal{J}_{\alpha \beta}^{i j}=\operatorname{Im}\left(U_{\alpha i} U_{\beta j} U_{\alpha j}^{*} U_{\beta i}^{*}\right)
$$

\footnotetext{
${ }^{2}$ The Feynman-'t Hooft gauge has been adopted in Ref. [30] for the calculations of radiative $\beta^{-} \rightarrow \alpha^{-}+\gamma$ decays, but In Ref. [31] the authors made no mention of which gauge has been used to carry out the Feynman-diagram calculations. Here we make use of the unitary gauge instead of other gauges, so as to minimize the number of Feynman diagrams and make an independent crosscheck of the results obtained in the literature.
} 


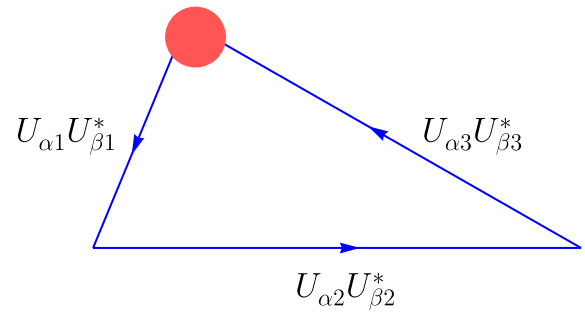

Fig. 1 The schematic plot of an effective Dirac-type unitarity triangle and four typical topologies of its apex in the complex plane based on the $(3+n)$ active-sterile neutrino mixing scheme, where $(\alpha, \beta)=(e, \mu)$, $(\mu, \tau)$ or $(\tau, e)$. As for the effective apex in red, pattern a corresponds to the exact unitarity triangle of three active neutrinos defined by Eq. (1); and pattern b1, b2 or $\mathbf{b 3}$ stands for a unitarity polygon of $(3+n)$ neutrinos defined by Eq. (3)

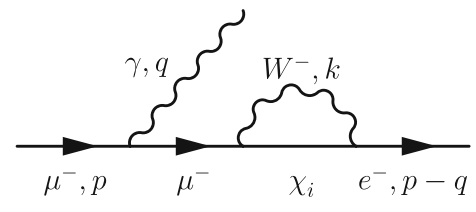

(a)

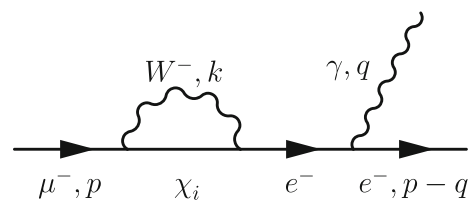

(b)

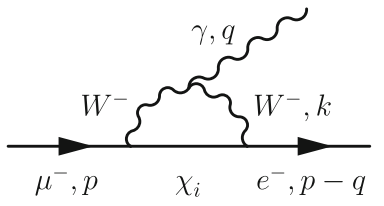

(c)

Fig. 2 The one-loop Feynman diagrams contributing to $\mu^{-} \rightarrow e^{-}+\gamma$ in the unitary gauge, where $\chi_{i}$ can be either three active neutrinos (i.e., $\chi_{i}=v_{i}$ with mass $m_{i}$ for $\left.i=1,2,3\right)$ or $n$ sterile neutrinos (i.e., $\chi_{i+3}=N_{i}$ with mass $M_{i}$ for $\left.i=1,2, \ldots, n\right)$ in the $(3+n)$ active-sterile flavor mixing scheme

where $(\alpha, \beta)=(e, \mu),(\mu, \tau)$ or $(\tau, e)$ and $(i, j)=$ $(1,2),(2,3)$ or $(3,1)$, and their differences signify slight unitarity violation of the $3 \times 3$ PMNS matrix $U$.

Therefore, we expect that the main results of our study will be useful for testing unitarity of the PMNS matrix and probing possible active-sterile neutrino mixing in the era of precision measurements of neutrino oscillations, charged lepton flavor violation and even lepton number violation.

The remaining parts of this paper are organized as follows. In Sect. 2 we calculate the rates of radiative $\beta^{-} \rightarrow \alpha^{-}+\gamma$ decays in the $(3+n)$ active-sterile neutrino mixing scheme by making use of the unitary gauge, and discuss some salient features of the loop function. Section 3 is devoted to some straightforward but strong constraints on a given unitarity polygons in a natural seesaw framework, and to some explicit discussions about its sub-triangles $\triangle_{\alpha \beta}^{i j}$ by taking the most popular $(3+3)$ active-sterile neutrino mixing scenario for example. We summarize our main results in Sect. 4.

\section{Radiative $\beta^{-} \rightarrow \alpha^{-}+\gamma$ decays}

Let us take $\mu^{-} \rightarrow e^{-}+\gamma$ as an example to show how to calculate the rates of radiative $\beta^{-} \rightarrow \alpha^{-}+\gamma$ decays for $(\alpha, \beta)=(e, \mu),(e, \tau)$ or $(\mu, \tau)$. Given the $(3+n)$ activesterile neutrino mixing scheme and the weak charged-current interactions of such neutrinos as described by Eq. (2), the lowest-order (one-loop) Feynman diagrams which contribute to $\mu^{-} \rightarrow e^{-}+\gamma$ in the unitary gauge are plotted in Fig. 2, where $\chi_{i}$ with mass $\lambda_{i}$ (for $i=1,2, \ldots, 3+n$ ) represents an arbitrary neutrino field under discussion no matter whether it is active or sterile and whether it is light or heavy. For the sake of simplicity, one may tentatively use $\mathcal{U}_{\alpha i}$ (for $i=$ $1,2, \ldots, 3+n$ ) to universally describe $U_{\alpha i}$ (for $i=1,2,3$ ) and $R_{\alpha i}$ (for $i=1,2, \ldots, n$ ), since $U$ and $R$ are respectively the upper-left $3 \times 3$ and upper-right $3 \times n$ sub-matrices of the $(3+n) \times(3+n)$ unitary matrix $\mathcal{U}$. With the help of the notations $\chi_{i}, \lambda_{i}$ and $\mathcal{U}_{\alpha i}$, the active and sterile neutrinos can be treated on the same footing in calculating the rate of $\mu^{-} \rightarrow e^{-}+\gamma$.

In the unitary gauge and $D$ dimensions $(D \equiv 4-\varepsilon)$, the three decay amplitudes for $\mu^{-} \rightarrow e^{-}+\gamma$ shown in Fig. 2 can be expressed as

$$
\begin{aligned}
\mathrm{i} \mathcal{M}_{\mathrm{a}}= & -\frac{1}{2} e^{2} \epsilon_{\rho}^{*}(q) \sum_{i=1}^{3+n} \mathcal{U}_{e i} \mathcal{U}_{\mu i}^{*} \mu^{\frac{3}{2} \varepsilon} \int \frac{\mathrm{d}^{D} k}{(2 \pi)^{D}} \bar{u}(p-q) \\
& \times \gamma^{\mu} P_{\mathrm{L}} \frac{\not p-q-\not k+\lambda_{i}}{(p-q-k)^{2}-\lambda_{i}^{2}} \gamma^{v} P_{\mathrm{L}} \\
& \times \frac{\not p-q+m_{\mu}}{(p-q)^{2}-m_{\mu}^{2}} \gamma^{\rho} u(p)\left(g_{\mu \nu}-\frac{k_{\mu} k_{v}}{M_{W}^{2}}\right) \frac{1}{k^{2}-M_{W}^{2}}, \\
\mathrm{i} \mathcal{M}_{\mathrm{b}}= & -\frac{1}{2} e g^{2} \epsilon_{\rho}^{*}(q) \sum_{i=1}^{3+n} \mathcal{U}_{e i} \mathcal{U}_{\mu i}^{*} \mu^{\frac{3}{2} \varepsilon} \int \frac{\mathrm{d}^{D} k}{(2 \pi)^{D}} \bar{u}(p-q) \\
& \times \gamma^{\rho} \frac{\not p+m_{e}}{p^{2}-m_{e}^{2}} \gamma^{\mu} P_{\mathrm{L}} \\
& \times \frac{\not p-\not k+\lambda_{i}}{(p-k)^{2}-\lambda_{i}^{2}} \gamma^{v} P_{\mathrm{L}} u(p)\left(g_{\mu \nu}-\frac{k_{\mu} k_{v}}{M_{W}^{2}}\right) \frac{1}{k^{2}-M_{W}^{2}},
\end{aligned}
$$




$$
\begin{aligned}
\mathrm{i} \mathcal{M}_{\mathrm{c}}= & \frac{1}{2} e g^{2} \epsilon_{\rho}^{*}(q) \sum_{i=1}^{3+n} \mathcal{U}_{e i} \mathcal{U}_{\mu i}^{*} \mu^{\frac{3}{2} \varepsilon} \int \frac{\mathrm{d}^{D} k}{(2 \pi)^{D}} \bar{u}(p-q) \\
& \times \gamma^{\mu} P_{\mathrm{L}} \frac{\not p-q-\not k+\lambda_{i}}{(p-q-k)^{2}-\lambda_{i}^{2}} \gamma^{\nu} P_{\mathrm{L}} u(p) \\
& \times\left(g_{\mu \sigma}-\frac{k_{\mu} k_{\sigma}}{M_{W}^{2}}\right) \frac{1}{k^{2}-M_{W}^{2}}\left[-g^{\sigma \lambda}(q+2 k)^{\rho}\right. \\
& \left.+g^{\lambda \rho}(2 q+k)^{\sigma}+g^{\rho \sigma}(k-q)^{\lambda}\right] \\
& \times\left[g_{\lambda \nu}-\frac{(q+k)_{\lambda}(q+k)_{v}}{M_{W}^{2}}\right] \frac{1}{(q+k)^{2}-M_{W}^{2}},
\end{aligned}
$$

where $\mu$ is an arbitrary mass-dimension-one parameter to keep the electromagnetic and weak coupling constants $e$ and $g$ to be dimensionless in $D$ dimensions. A more specific introduction of $\mu$, together with the algebra and traces of the Dirac matrices in $D$ dimensions, has been given in appendix $\mathrm{A}$. In appendix B we have explicitly figured out the integrals in Eq. (5) with the help of the on-shell conditions $p^{2}=m_{\mu}^{2}$, $q^{2}=0$ and $p \cdot q=\left(m_{\mu}^{2}-m_{e}^{2}\right) / 2$, the physical polarizations for the external photon $\epsilon(q) \cdot q=0$ and the well-known Passarino-Veltman integrals. Then we take the limit $D \rightarrow 4$ (i.e., $\varepsilon \rightarrow 0$ ) and arrive at the results

$$
\begin{aligned}
\mathrm{i} \mathcal{M}_{\mathrm{a}}= & \frac{\mathrm{i} e g^{2}}{2(4 \pi)^{2}} \epsilon_{\rho}^{*}(q) \bar{u}(p-q) \frac{m_{e}\left(m_{e} P_{\mathrm{R}}+m_{\mu} P_{\mathrm{L}}\right)}{m_{\mu}^{2}-m_{e}^{2}} \gamma^{\rho} u(p) \\
& \times \sum_{i=1}^{3+n} \mathcal{U}_{e i} \mathcal{U}_{\mu i}^{*} \mu^{\frac{1}{2} \varepsilon} \\
& \times\left\{\frac{1}{2}\left(3 x_{i}-\frac{m_{e}^{2}}{M_{W}^{2}}\right)\left(\Delta-\ln \frac{M_{W}^{2}}{\mu^{2}}\right)\right. \\
& +\frac{5 x_{i}^{2}-5 x_{i}-6}{4\left(x_{i}-1\right)}-\frac{3 x_{i}^{2}\left(x_{i}-2\right)}{2\left(x_{i}-1\right)^{2}} \ln x_{i} \\
& -\frac{m_{e}^{2}}{M_{W}^{2}}\left[\frac{x_{i}^{3}-3 x_{i}^{2}+45 x_{i}-7}{12\left(x_{i}-1\right)^{3}}\right. \\
& \left.\left.-\frac{x_{i}^{2}\left(x_{i}^{2}-4 x_{i}+9\right)}{2\left(x_{i}-1\right)^{4}} \ln x_{i}\right]\right\}
\end{aligned}
$$

and

$$
\begin{aligned}
\mathrm{i} \mathcal{M}_{\mathrm{b}}= & -\frac{\mathrm{i} e g^{2}}{2(4 \pi)^{2}} \epsilon_{\rho}^{*}(q) \bar{u}(p-q) \\
& \times \frac{m_{\mu}\left(m_{\mu} P_{\mathrm{R}}+m_{e} P_{\mathrm{L}}\right)}{m_{\mu}^{2}-m_{e}^{2}} \gamma^{\rho} u(p) \sum_{i=1}^{3+n} \mathcal{U}_{e i} \mathcal{U}_{\mu i}^{*} \mu^{\frac{1}{2} \varepsilon} \\
& \times\left\{\frac{1}{2}\left(3 x_{i}-\frac{m_{\mu}^{2}}{M_{W}^{2}}\right)\left(\Delta-\ln \frac{M_{W}^{2}}{\mu^{2}}\right)\right. \\
& +\frac{5 x_{i}^{2}-5 x_{i}-6}{4\left(x_{i}-1\right)}-\frac{3 x_{i}^{2}\left(x_{i}-2\right)}{2\left(x_{i}-1\right)^{2}} \ln x_{i} \\
& -\frac{m_{\mu}^{2}}{M_{W}^{2}}\left[\frac{x_{i}^{3}-3 x_{i}^{2}+45 x_{i}-7}{12\left(x_{i}-1\right)^{3}}\right.
\end{aligned}
$$

$$
\left.\left.-\frac{x_{i}^{2}\left(x_{i}^{2}-4 x_{i}+9\right)}{2\left(x_{i}-1\right)^{4}} \ln x_{i}\right]\right\},
$$

as well as

$$
\begin{aligned}
\mathrm{i} \mathcal{M}_{\mathrm{c}}= & -\frac{\mathrm{i} e g^{2}}{2(4 \pi)^{2} M_{W}^{2}} \epsilon_{\rho}^{*}(q) \sum_{i=1}^{3+n} \mathcal{U}_{e i} \mathcal{U}_{\mu i}^{*} \mu^{\frac{1}{2} \varepsilon} \\
& \times\left\{G_{\gamma}\left(x_{i}\right) \bar{u}(p-q) \mathrm{i} \sigma^{\rho \lambda} q_{\lambda}\left(m_{e} P_{\mathrm{L}}+m_{\mu} P_{\mathrm{R}}\right) u(p)\right. \\
& +\bar{u}(p-q)\left[m_{e} m_{\mu} P_{\mathrm{L}}+\left(m_{e}^{2}+m_{\mu}^{2}\right) P_{\mathrm{R}}\right] \gamma^{\rho} u(p) \\
& \times\left[\frac{x_{i}^{3}-3 x_{i}^{2}+45 x_{i}-7 x_{i}^{2}\left(x_{i}^{2}-4 x_{i}+9\right)}{\left.2\left(x_{i}-1\right)^{3} \ln x_{i}\right]}\right. \\
& -\bar{u}(p-q) P_{\mathrm{R}} \gamma^{\rho} u(p) M_{W}^{2}\left[\frac{5 x_{i}^{2}-5 x_{i}-6}{4\left(x_{i}-1\right)}\right. \\
& \left.-\times \frac{3 x_{i}^{2}\left(x_{i}-2\right)}{2\left(x_{i}-1\right)^{2}} \ln x_{i}\right]+\frac{1}{2} \bar{u}(p-q)\left[m_{e} m_{\mu} P_{\mathrm{L}}\right. \\
& \left.+\left(m_{e}^{2}+m_{\mu}^{2}-3 x_{i} M_{W}^{2}\right) P_{\mathrm{R}}\right] \gamma^{\rho} u(p) \\
& \left.\times\left(\Delta-\ln \frac{M_{W}^{2}}{\mu^{2}}\right)\right\}
\end{aligned}
$$

where $x_{i} \equiv \lambda_{i}^{2} / M_{W}^{2}, \Delta \equiv 2 / \varepsilon-\gamma_{\mathrm{E}}+\ln (4 \pi)$ with $\gamma_{\mathrm{E}}$ being Euler's constant, and

$G_{\gamma}\left(x_{i}\right)=-\frac{5}{6}-\frac{2 x_{i}^{3}+5 x_{i}^{2}-x_{i}}{4\left(1-x_{i}\right)^{3}}-\frac{3 x_{i}^{3}}{2\left(1-x_{i}\right)^{4}} \ln x_{i}$

Note that both i $\mathcal{M}_{a}+\mathrm{i} \mathcal{M}_{b}$ and i $\mathcal{M}_{c}$ keep unchanged under the exchange $m_{e} \leftrightarrow m_{\mu}$, and the former can be explicitly expressed as

$$
\begin{aligned}
\mathrm{i} \mathcal{M}_{a} & +\mathrm{i} \mathcal{M}_{b} \\
= & \frac{\mathrm{i} e g^{2}}{2(4 \pi)^{2} M_{W}^{2}} \epsilon_{\rho}^{*}(q) \sum_{i=1}^{3+n} \mathcal{U}_{e i} \mathcal{U}_{\mu i}^{*} \mu^{\frac{1}{2} \varepsilon} \\
& \times\left\{\bar{u}(p-q)\left[m_{e} m_{\mu} P_{\mathrm{L}}+\left(m_{e}^{2}+m_{\mu}^{2}\right) P_{\mathrm{R}}\right] \gamma^{\rho} u(p)\right. \\
& \times\left[\frac{x_{i}^{3}-3 x_{i}^{2}+45 x_{i}-7}{12\left(x_{i}-1\right)^{3}}-\frac{x_{i}^{2}\left(x_{i}^{2}-4 x_{i}+9\right)}{2\left(x_{i}-1\right)^{4}} \ln x_{i}\right] \\
& -\bar{u}(p-q) P_{\mathrm{R}} \gamma^{\rho} u(p) M_{W}^{2} \\
\times & {\left[\frac{5 x_{i}^{2}-5 x_{i}-6}{4\left(x_{i}-1\right)}-\frac{3 x_{i}^{2}\left(x_{i}-2\right)}{2\left(x_{i}-1\right)^{2}} \ln x_{i}\right] } \\
+ & \frac{1}{2} \bar{u}(p-q)\left[m_{e} m_{\mu} P_{\mathrm{L}}\right. \\
+ & \left.\left.\left(m_{e}^{2}+m_{\mu}^{2}-3 x_{i} M_{W}^{2}\right) P_{\mathrm{R}}\right] \gamma^{\rho} u(p)\left(\Delta-\ln \frac{M_{W}^{2}}{\mu^{2}}\right)\right\}
\end{aligned}
$$


It becomes clear that the terms of $\mathrm{i}_{a}+\mathrm{i} \mathcal{M}_{b}$ in Eq. (10) can exactly eliminate the terms of $\mathrm{i} \mathcal{M}_{c}$ shown in the last four rows of Eq. (8), including the divergent terms and $\mu$-dependent terms. This observation means that it is unnecessary to invoke any specific renormalization scheme to assure the total decay amplitude $\mathcal{M}=\mathcal{M}_{\mathrm{a}}+\mathcal{M}_{\mathrm{b}}+\mathcal{M}_{\mathrm{c}}$ to be finite, and thus the overall factor $\mu^{\varepsilon / 2}$ can be simply removed from $\mathcal{M}$. As a result,

$$
\begin{aligned}
\mathrm{i} \mathcal{M}= & \frac{-\mathrm{i} e g^{2}}{2(4 \pi)^{2} M_{W}^{2}} \sum_{i=1}^{3+n} \mathcal{U}_{e i} \mathcal{U}_{\mu i}^{*} G_{\gamma}\left(x_{i}\right) \\
& {\left[\epsilon_{\rho}^{*}(q) \bar{u}(p-q) \mathrm{i} \sigma^{\rho \lambda} q_{\lambda}\left(m_{e} P_{\mathrm{L}}+m_{\mu} P_{\mathrm{R}}\right) u(p)\right] . }
\end{aligned}
$$

We conclude that our results in Eqs. (9) and (11) are consistent with those obtained in Refs. [29-31,42-45], but some necessary comments and clarifications are in order.

- In Ref. [42] a similar loop function $F\left(x_{i}\right)$ for $\mu^{-} \rightarrow e^{-}+$ $\gamma$ has been obtained in the canonical seesaw framework, but it is presented in the form of several integrals. After explicitly figuring out those integrals, we arrive at

$$
F\left(x_{i}\right)=\frac{10}{3}+\frac{2 x_{i}^{3}+5 x_{i}^{2}-x_{i}}{\left(1-x_{i}\right)^{3}}+\frac{6 x_{i}^{3}}{\left(1-x_{i}\right)^{4}} \ln x_{i} .
$$

It is clear that $G_{\gamma}\left(x_{i}\right)=-F\left(x_{i}\right) / 4$ holds, and the factor $-1 / 4$ can be compensated by an additional factor -4 in the total decay amplitude $\mathcal{M}$ of ours. Therefore, our result is fully in agreement with the one obtained in Ref. [42].

- Note that the constant in either $G_{\gamma}\left(x_{i}\right)$ or $F\left(x_{i}\right)$ can be exactly eliminated due to the unitarity of $\mathcal{U}$ in the $(3+n)$ active-sterile neutrino mixing scheme. That is why a result which is equivalent to $-\left[F\left(x_{i}\right)-10 / 3\right]$ has been given in Ref. [45]. If one is subject to the minimal unitarity violation scheme as considered in Refs. [38,39], the calculations and results shown in Eqs. (5)-(11) remain valid after $n=0$ and $\mathcal{U}=U$ are taken; but one should keep in mind that in this case $\mathcal{U}=U$ is not unitary due to the influence of one or more dimension- 6 operators, and the corresponding loop function is actually $F\left(x_{i}\right)$ in Eq. (12) after $\mathcal{M}$ is replaced by $-\mathcal{M} / 4$. Hence the constant 10/3 in $F\left(x_{i}\right)$ cannot be eliminated because of the non-unitarity of $U$, and it will be the dominant part of $F\left(x_{i}\right)$ contributing to the total decay amplitude of $\mu^{-} \rightarrow e^{-}+\gamma$. This point deserves to be highlighted, so as to distinguish the more phenomenological minimal unitarity violation case from a generic $(3+n)$ activesterile neutrino mixing case [46].

- In view of Refs. [43,44], where the radiative decays of quarks are calculated at the one-loop level, we find that a similar loop function can be extracted after we switch

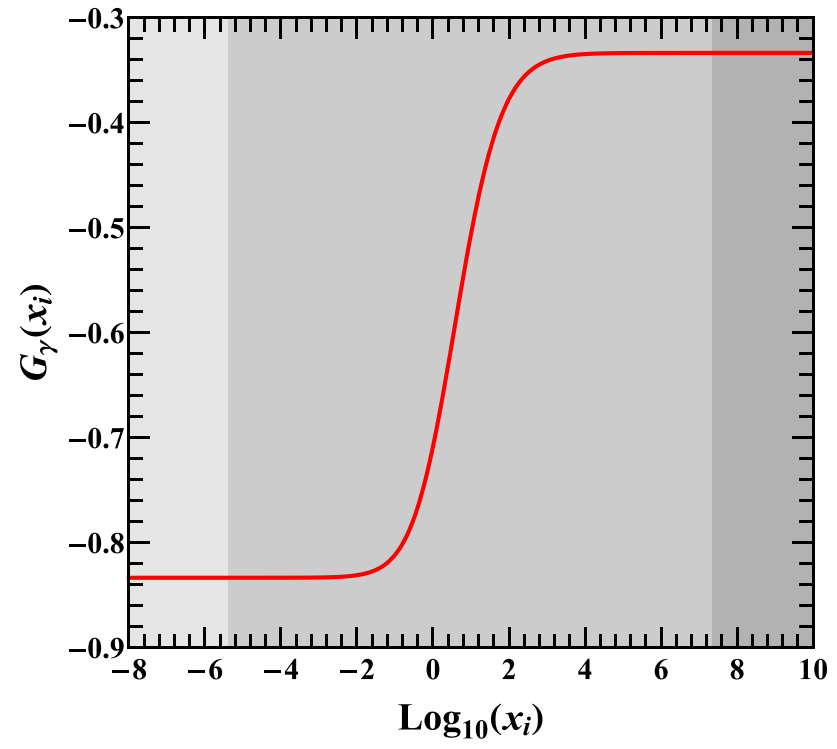

Fig. 3 A numerical illustration of the loop function $G_{\gamma}\left(x_{i}\right)$ evolving with $x_{i} \equiv \lambda_{i}^{2} / M_{W}^{2}$. In the light gray $\left(x_{i} \lesssim 4.0 \times 10^{-6}\right)$ and dark gray $\left(x_{i} \gtrsim 2.2 \times 10^{7}\right)$ regimes, the asymptotic limits $G_{\gamma}\left(x_{i}\right)=-5 / 6$ and $-1 / 3$ hold respectively up to the accuracy of $\mathcal{O}\left(10^{-6}\right)$

off the contributions of those extra Feynman diagrams which are irrelevant to Fig. 2:

$\widetilde{F}\left(x_{i}\right)=-\frac{2 x_{i}^{3}+5 x_{i}^{2}-x_{i}}{4\left(1-x_{i}\right)^{3}}-\frac{3 x_{i}^{3}}{2\left(1-x_{i}\right)^{4}} \ln x_{i}$.

This result is exactly the loop function of $\mu^{-} \rightarrow e^{-}+\gamma$ that has been obtained in Refs. [29-31]. It is obvious that the results given in Eqs. (9) and (13) differ from each other by a constant $-5 / 6$; namely, $G_{\gamma}\left(x_{i}\right)=\widetilde{F}\left(x_{i}\right)-5 / 6$. As discussed above, this constant can be eliminated when the unitarity of $\mathcal{U}$ is taken into consideration.

In short, one should be careful in adopting an explicit expression for the loop function of radiative decays of charged leptons when discussing the non-unitarity of the PMNS matrix $U$, because the issue depends on which unitarity violation scheme is under discussion.

Since the form of $G_{\gamma}\left(x_{i}\right)$ itself is universal for both active and sterile neutrinos, it is very useful to consider its behaviors evolving with $x_{i}$, especially in the extreme cases $x_{i} \ll 1$ (i.e., $\lambda_{i}^{2} \ll M_{W}^{2}$ ) and $x_{i} \gg 1$ (i.e., $\lambda_{i}^{2} \gg M_{W}^{2}$ ). One can easily observe $G_{\gamma}\left(x_{i}\right)=-5 / 6$ for $x_{i} \ll 1$ and $G_{\gamma}\left(x_{i}\right)=-1 / 3$ for $x_{i} \gg 1$, as numerically illustrated in Fig. 3. In the regions of $x_{i} \lesssim 4.0 \times 10^{-6}$ (light gray) and $x_{i} \gtrsim 2.2 \times 10^{7}$ (dark gray), or equivalently $\lambda_{i} \lesssim 0.16 \mathrm{GeV}$ and $\lambda_{i} \gtrsim 3.8 \times 10^{5}$ $\mathrm{GeV}$, we find that $G_{\gamma}\left(x_{i}\right)+5 / 6<10^{-6}$ and $-G_{\gamma}\left(x_{i}\right)-$ $1 / 3<10^{-6}$ hold, respectively. These two thresholds may be used to roughly define the regimes of "light" and "heavy" sterile neutrinos when dealing with the contributions of such 


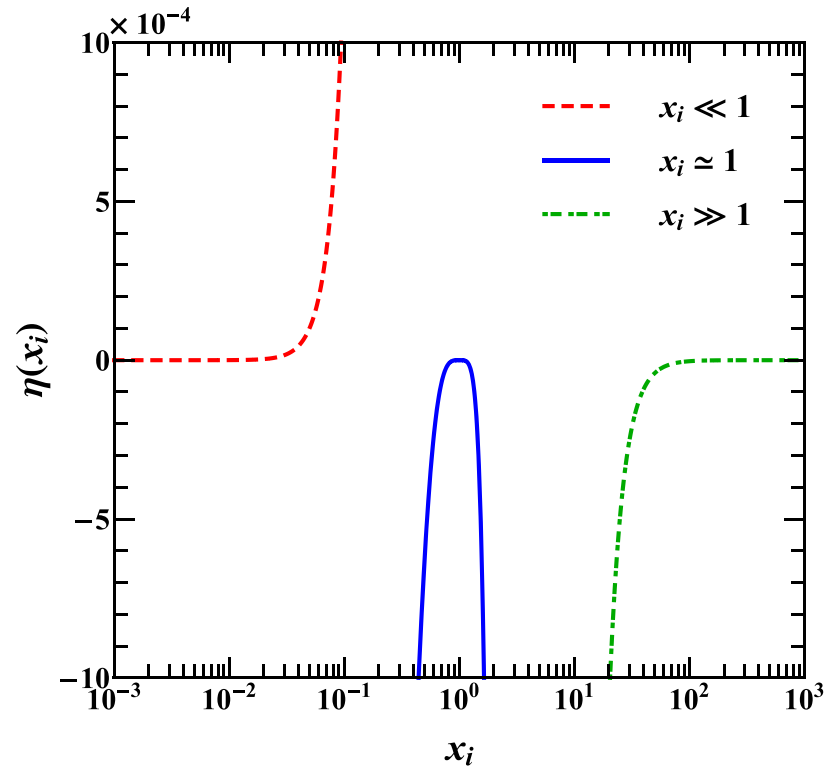

Fig. 4 A numerical illustration of the relative error $\eta\left(x_{i}\right)$ between the exact result of $G_{\gamma}\left(x_{i}\right)$ and the analytical approximations given in Eq. (14), in which $x_{i} \lesssim 0.1,0.1 \lesssim x_{i} \lesssim 10$ and $x_{i} \gtrsim 10$ have been taken to plot $\eta\left(x_{i}\right)$ corresponding to the regimes of $x_{i} \ll 1, x_{i} \simeq 1$ and $x_{i} \gg 1$, respectively

new degrees of freedom to the loop function of radiative $\beta^{-} \rightarrow \alpha^{-}+\gamma$ decays for $(\alpha, \beta)=(e, \mu),(e, \tau)$ and $(\mu, \tau)$.

On the other hand, it is also instructive and helpful to make an analytical approximation for the expression of $G_{\gamma}\left(x_{i}\right)$ in three typical regions of $x_{i}$ (i.e., $x_{i} \ll 1, x_{i} \simeq 1$ and $x_{i} \gg 1$ ). To a good degree of accuracy, we obtain the approximate analytical results
We proceed to calculate the unpolarized decay rate of $\mu^{-} \rightarrow e^{-}+\gamma$ by using the formula

$$
\begin{aligned}
\Gamma\left(\mu^{-} \rightarrow e^{-}+\gamma\right)= & \frac{1}{2 m_{\mu}} \cdot \frac{1}{8 \pi}\left(1-\frac{m_{e}^{2}}{m_{\mu}^{2}}\right) \\
& \cdot \frac{1}{2} \sum|\mathcal{M}|^{2},
\end{aligned}
$$

in which the last part means that $|\mathcal{M}|^{2}$ is averaged over the spin states of $\mu^{-}$and summed over the spin states of $e^{-}$and the polarization states of $\gamma$. To be explicit,

$$
\begin{aligned}
\frac{1}{2} \sum|\mathcal{M}|^{2}= & -\frac{e^{2} g^{4}}{8(4 \pi)^{4} M_{W}^{4}}\left|\sum_{i=1}^{3+n} \mathcal{U}_{e i} \mathcal{U}_{\mu i}^{*} G_{\gamma}\left(x_{i}\right)\right|^{2} q_{\lambda} q_{\mu} \\
& \operatorname{Tr}\left[\left(\not p-q+m_{e}\right) \sigma^{\rho \lambda}\left(m_{e} P_{\mathrm{L}}+m_{\mu} P_{\mathrm{R}}\right)\right. \\
& \left.\times\left(\not p+m_{\mu}\right)\left(m_{e} P_{\mathrm{R}}+m_{\mu} P_{\mathrm{L}}\right) \sigma_{\rho}^{\mu}\right] \\
= & \frac{e^{2} g^{4}}{4(4 \pi)^{4} M_{W}^{4}}\left(m_{\mu}^{2}+m_{e}^{2}\right)\left(m_{\mu}^{2}-m_{e}^{2}\right)^{2} \\
& \left|\sum_{i=1}^{3+n} \mathcal{U}_{e i} \mathcal{U}_{\mu i}^{*} G_{\gamma}\left(x_{i}\right)\right|^{2}
\end{aligned}
$$

As a result,

$$
\begin{aligned}
\Gamma\left(\mu^{-} \rightarrow e^{-}+\gamma\right) \\
=\frac{\alpha_{\mathrm{em}} G_{\mathrm{F}}^{2} m_{\mu}^{5}}{128 \pi^{4}}\left(1+\frac{m_{e}^{2}}{m_{\mu}^{2}}\right)\left(1-\frac{m_{e}^{2}}{m_{\mu}^{2}}\right)^{3} \\
\quad \times\left|\sum_{i=1}^{3+n} \mathcal{U}_{e i} \mathcal{U}_{\mu i}^{*} G_{\gamma}\left(x_{i}\right)\right|^{2},
\end{aligned}
$$

The relative error between such analytical approximations and the exact result of $G_{\gamma}\left(x_{i}\right)$, denoted as $\eta\left(x_{i}\right) \equiv$ $\left[\widetilde{G}_{\gamma}\left(x_{i}\right)-G_{\gamma}\left(x_{i}\right)\right] / G_{\gamma}\left(x_{i}\right)$, is numerically illustrated in Fig. 4 , where $x_{i} \lesssim 0.1,0.1 \lesssim x_{i} \lesssim 10$ and $x_{i} \gtrsim 10$ have been taken to plot $\eta\left(x_{i}\right)$ corresponding to the ranges of $x_{i} \ll 1, x_{i} \simeq 1$ and $x_{i} \gg 1$, respectively. As can be seen in Fig. $4, \eta\left(x_{i}\right) \lesssim 1 \times 10^{-4}$ holds in the regions of $x_{i} \lesssim 0.05,0.67 \lesssim x_{i} \lesssim 1.36$ and $x_{i} \gtrsim 38$. This means that in such regions the analytical approximations made in Eq. (14) coincide with the exact result of $G_{\gamma}\left(x_{i}\right)$ very well. In particular, the masses of heavy Majorana neutrinos in the canonical seesaw mechanism does satisfy $x_{i} \gg 1$. (for $\left.x_{i} \ll 1\right)$,

(for $\left.x_{i} \simeq 1\right)$,

(for $x_{i} \gg 1$ ). where $\alpha_{\mathrm{em}} \equiv e^{2} /(4 \pi)$ is the fine-structure constant of electromagnetic interactions, and $G_{\mathrm{F}} \equiv g^{2} /\left(4 \sqrt{2} M_{W}^{2}\right)$ is the Fermi coupling constant of weak interactions.

Extending the above calculations to the radiative decays $\tau^{-} \rightarrow e^{-}+\gamma$ and $\tau^{-} \rightarrow \mu^{-}+\gamma$, one may directly write out the unpolarized decay rate of $\beta^{-} \rightarrow \alpha^{-}+\gamma$ as follows:

$$
\begin{aligned}
\Gamma\left(\beta^{-} \rightarrow \alpha^{-}+\gamma\right)= & \frac{\alpha_{\mathrm{em}} G_{\mathrm{F}}^{2} m_{\beta}^{5}}{128 \pi^{4}}\left(1+\frac{m_{\alpha}^{2}}{m_{\beta}^{2}}\right)\left(1-\frac{m_{\alpha}^{2}}{m_{\beta}^{2}}\right)^{3} \\
& \times\left|\sum_{i=1}^{3+n} \mathcal{U}_{\alpha i} \mathcal{U}_{\beta i}^{*} G_{\gamma}\left(x_{i}\right)\right|^{2},
\end{aligned}
$$


where $(\alpha, \beta)=(e, \mu),(e, \tau)$ or $(\mu, \tau)$. In comparison, the pure leptonic decays $\beta^{-} \rightarrow \alpha^{-}+\bar{v}_{\alpha}+v_{\beta}$ are also mediated by $W^{-}$and their rates in the $(3+n)$ active-sterile neutrino mixing scheme are given by

$$
\begin{aligned}
\Gamma & \left(\beta^{-} \rightarrow \alpha^{-}+\bar{v}_{\alpha}+v_{\beta}\right) \\
= & \frac{G_{\mathrm{F}}^{2} m_{\beta}^{5}}{192 \pi^{3}}\left(1-8 \frac{m_{\alpha}^{2}}{m_{\beta}^{2}}\right)\left[1+\frac{\alpha_{\mathrm{em}}}{2 \pi}\left(\frac{25}{4}-\pi^{2}\right)\right] \\
& \times \sum_{i=1}^{3} \sum_{j=1}^{3}\left|\mathcal{U}_{\alpha i}\right|^{2}\left|\mathcal{U}_{\beta j}\right|^{2},
\end{aligned}
$$

in which the term proportional to $\alpha_{\mathrm{em}}$ stands for the electromagnetic corrections [29]. Note that

$$
\begin{gathered}
\sum_{i=1}^{3} \sum_{j=1}^{3}\left|\mathcal{U}_{\alpha i}\right|^{2}\left|\mathcal{U}_{\beta j}\right|^{2}=\sum_{i=1}^{3} \sum_{j=1}^{3}\left|U_{\alpha i}\right|^{2}\left|U_{\beta j}\right|^{2} \\
=\left(1-\sum_{i=1}^{n}\left|R_{\alpha i}\right|^{2}\right)\left(1-\sum_{j=1}^{n}\left|R_{\beta j}\right|^{2}\right),
\end{gathered}
$$

where $U U^{\dagger}+R R^{\dagger}=I$ has been used, and thus it is expected to be only slightly departure from one. It is therefore straightforward to obtain the dimensionless ratio

$$
\begin{aligned}
\xi\left(\beta^{-} \rightarrow \alpha^{-}+\gamma\right) \equiv & \frac{\Gamma\left(\beta^{-} \rightarrow \alpha^{-}+\gamma\right)}{\Gamma\left(\beta^{-} \rightarrow \alpha^{-}+\bar{v}_{\alpha}+v_{\beta}\right)} \\
\simeq & \frac{3 \alpha_{\mathrm{em}}}{2 \pi} \mid \sum_{i=1}^{3} U_{\alpha i} U_{\beta i}^{*} G_{\gamma}\left(\frac{m_{i}^{2}}{M_{W}^{2}}\right) \\
& +\left.\sum_{i=1}^{n} R_{\alpha i} R_{\beta i}^{*} G_{\gamma}\left(\frac{M_{i}^{2}}{M_{W}^{2}}\right)\right|^{2},
\end{aligned}
$$

in the leading-order approximation by neglecting those next-to-leading-order and higher-order contributions. Taking account of the asymptotic behaviors of the loop function shown above, we are going to discuss how to constrain the unitarity polygons by means of the experimental upper bounds of $\xi\left(\beta^{-} \rightarrow \alpha^{-}+\gamma\right)$.

\section{Constraints on the unitarity polygons}

In the $(3+n)$ active-sterile neutrino mixing scheme, it is natural to assume that the $n$ sterile neutrinos are heavy enough such that the seesaw mechanism takes effect to explain why three active neutrinos are so light. Given this well-motivated picture with $M_{i} \gg M_{W}$, one may accordingly simplify the loop functions $G_{\gamma}\left(m_{i}^{2} / M_{W}^{2}\right)$ and $G_{\gamma}\left(M_{i}^{2} / M_{W}^{2}\right)$. Taking account of the analytical approximations made in Eq. (14) and the unitarity condition $U U^{\dagger}+R R^{\dagger}=I$, we obtain

$$
\begin{aligned}
& \xi\left(\beta^{-} \rightarrow \alpha^{-}+\gamma\right) \\
& \simeq \frac{3 \alpha_{\mathrm{em}}}{2 \pi}\left|\sum_{i=1}^{3} U_{\alpha i} U_{\beta i}^{*}\left(-\frac{5}{6}+\frac{1}{4} \cdot \frac{m_{i}^{2}}{M_{W}^{2}}\right)-\frac{1}{3} \sum_{i=1}^{n} R_{\alpha i} R_{\beta i}^{*}\right|^{2} \\
& =\frac{3 \alpha_{\mathrm{em}}}{8 \pi}\left|\sum_{i=1}^{3} U_{\alpha i} U_{\beta i}^{*}\left(1-\frac{1}{2} \cdot \frac{m_{i}^{2}}{M_{W}^{2}}\right)\right|^{2}
\end{aligned}
$$

up to the leading order of $R_{\alpha i} R_{\beta i}^{*}$ (for $i=1,2, \ldots, n$ ). Two immediate observations are in order.

- If all the new degrees of freedom are switched off, the PMNS matrix $U$ is exactly unitary and thus one arrives at the "standard" result

$$
\begin{aligned}
\xi\left(\beta^{-} \rightarrow \alpha^{-}+\gamma\right) & \simeq \frac{3 \alpha_{\mathrm{em}}}{32 \pi}\left|\sum_{i=1}^{3} U_{\alpha i} U_{\beta i}^{*} \frac{m_{i}^{2}}{M_{W}^{2}}\right|^{2} \\
& =\frac{3 \alpha_{\mathrm{em}}}{32 \pi}\left|\sum_{i=2}^{3} U_{\alpha i} U_{\beta i}^{*} \frac{\Delta m_{i 1}^{2}}{M_{W}^{2}}\right|^{2} \\
& \lesssim \mathcal{O}\left(10^{-54}\right),
\end{aligned}
$$

where $\Delta m_{i 1}^{2} \equiv m_{i}^{2}-m_{1}^{2}$ is defined (for $i=2,3$ ), and current neutrino oscillation data [1] have been taken into account in making the above estimate [2]. In this case it is therefore hopeless to measure any radiative decays of charged leptons.

- If the PMNS matrix $U$ is not exactly unitary due to the existence of slight mixing between three active neutrinos and $n$ sterile heavy neutrinos as discussed above, then it is quite safe to neglect the $m_{i}^{2} / M_{W}^{2}$ terms in Eq. (22) and obtain a straightforward but strong constraint on the effective apex of a given effective Dirac-type unitarity triangle shown in Fig. 1:

$$
\begin{aligned}
\left|\sum_{i=1}^{3} U_{\alpha i} U_{\beta i}^{*}\right| & =\left|\sum_{i=1}^{n} R_{\alpha i} R_{\beta i}^{*}\right| \\
& =\sqrt{\frac{8 \pi}{3 \alpha_{\mathrm{em}}} \xi\left(\beta^{-} \rightarrow \alpha^{-}+\gamma\right)} \\
& \simeq 33.88 \sqrt{\xi\left(\beta^{-} \rightarrow \alpha^{-}+\gamma\right)}
\end{aligned}
$$

where $\alpha_{\mathrm{em}} \simeq 1 / 137$ has been input at low energies.

So Eq. (24) provides us with a realistic way to probe or constrain the seesaw-induced unitarity violation in radiative $\beta^{-} \rightarrow \alpha^{-}+\gamma$ decays (see also Ref. [46] for some similar discussions and observations). It is worth remarking that the coefficient in front of $\sqrt{\xi\left(\beta^{-} \rightarrow \alpha^{-}+\gamma\right)}$ under discussion differs from that obtained in the minimal unitarity 
violation scheme by a factor $5 / 3$ (i.e., the latter amounts to $\left.\sqrt{24 \pi /\left(25 \alpha_{\mathrm{em}}\right)} \simeq 20.33[38-40]\right)$.

To illustrate, let us take into account current experimental upper bounds on the branching fractions of $\beta^{-} \rightarrow \alpha^{-}+\gamma$ decays and $\beta^{-} \rightarrow \alpha^{-}+\bar{v}_{\alpha}+v_{\beta}$ decays [1]. That is,

$\mathcal{B}\left(\mu^{-} \rightarrow e^{-}+\gamma\right)<4.2 \times 10^{-13}$,

$\mathcal{B}\left(\tau^{-} \rightarrow e^{-}+\gamma\right)<3.3 \times 10^{-8}$,

$\mathcal{B}\left(\tau^{-} \rightarrow \mu^{-}+\gamma\right)<4.4 \times 10^{-8}$,

at the $90 \%$ confidence level, together with $\mathcal{B}\left(\mu^{-} \rightarrow e^{-}+\right.$ $\left.\bar{v}_{e}+v_{\mu}\right) \simeq 100 \%, \mathcal{B}\left(\tau^{-} \rightarrow e^{-}+\bar{v}_{e}+v_{\tau}\right) \simeq 17.82 \%$ and $\mathcal{B}\left(\tau^{-} \rightarrow \mu^{-}+\bar{v}_{\mu}+v_{\tau}\right) \simeq 17.39 \%$. Then we obtain the ratios

$\xi\left(\mu^{-} \rightarrow e^{-}+\gamma\right)<4.20 \times 10^{-13}$,

$\xi\left(\tau^{-} \rightarrow e^{-}+\gamma\right)<1.85 \times 10^{-7}$,

$\xi\left(\tau^{-} \rightarrow \mu^{-}+\gamma\right)<2.53 \times 10^{-7}$.

A combination of Eqs. (24) and (26) leads us to the constraints

$$
\begin{aligned}
\left|\sum_{i=1}^{3} U_{e i} U_{\mu i}^{*}\right| & =\left|\sum_{i=1}^{n} R_{e i} R_{\mu i}^{*}\right|<2.20 \times 10^{-5}, \\
\left|\sum_{i=1}^{3} U_{e i} U_{\tau i}^{*}\right| & =\left|\sum_{i=1}^{n} R_{e i} R_{\tau i}^{*}\right|<1.46 \times 10^{-2}, \\
\left|\sum_{i=1}^{3} U_{\mu i} U_{\tau i}^{*}\right| & =\left|\sum_{i=1}^{n} R_{\mu i} R_{\tau i}^{*}\right|<1.70 \times 10^{-2} .
\end{aligned}
$$

These results clearly show that the unitarity polygons in Fig. 1 can be treated as the effective unitarity triangles, since their differences are at most at the $\mathcal{O}\left(10^{-2}\right)$ level.

Now we take a brief look at the uncertainties induced by the approximations made to obtain Eq. (24). If the next-toleading-order terms of $G_{\gamma}\left(m_{i}^{2} / M_{W}^{2}\right)$ and $G_{\gamma}\left(M_{i}^{2} / M_{W}^{2}\right)$ are both taken into account, Eq. (22) will be replaced with

$$
\begin{aligned}
\xi\left(\beta^{-} \rightarrow \alpha^{-}+\gamma\right) & \simeq \frac{3 \alpha_{\mathrm{em}}}{2 \pi} \mid \sum_{i=1}^{3} U_{\alpha i} U_{\beta i}^{*}\left(-\frac{5}{6}+\frac{1}{4} \cdot \frac{m_{i}^{2}}{M_{W}^{2}}\right) \\
& +\left.\sum_{i=1}^{n} R_{\alpha i} R_{\beta i}^{*}\left[-\frac{1}{3}+\frac{1}{4} \cdot \frac{M_{W}^{2}}{M_{i}^{2}}\left(11+6 \ln \frac{M_{W}^{2}}{M_{i}^{2}}\right)\right]\right|^{2} \\
\simeq & \frac{3 \alpha_{\mathrm{em}}}{8 \pi} \mid \sum_{i=1}^{3} U_{\alpha i} U_{\beta i}^{*}-\frac{1}{2}\left[\sum_{i=1}^{3} U_{\alpha i} U_{\beta i}^{*} \frac{m_{i}^{2}}{M_{W}^{2}}\right. \\
& \left.+\sum_{i=1}^{n} R_{\alpha i} R_{\beta i}^{*} \frac{M_{W}^{2}}{M_{i}^{2}}\left(11+6 \ln \frac{M_{W}^{2}}{M_{i}^{2}}\right)\right]\left.\right|^{2}
\end{aligned}
$$

Making use of Eq. (28) and the formula ||$a|-| b|| \leq$ $|a+b| \leq|a|+|b|$ for two arbitrary complex quantities $a$ and $b$, one may estimate small corrections to the leading-order result given in Eq. (24):

$$
\begin{aligned}
\left|\sum_{i=1}^{3} U_{\alpha i} U_{\beta i}^{*}\right| \simeq & 33.88 \sqrt{\xi\left(\beta^{-} \rightarrow \alpha^{-}+\gamma\right)} \\
& \pm \frac{1}{2}\left[\sum_{i=1}^{3}\left|U_{\alpha i} U_{\beta i}^{*} \frac{m_{i}^{2}}{M_{W}^{2}}\right|\right. \\
& \left.+\sum_{i=1}^{n}\left|R_{\alpha i} R_{\beta i}^{*} \frac{M_{W}^{2}}{M_{i}^{2}}\left(11+6 \ln \frac{M_{W}^{2}}{M_{i}^{2}}\right)\right|\right],
\end{aligned}
$$

where the next-to-leading-order contributions have been treated as the uncertainties. Taking account of $U_{\alpha i} \sim 1$, $R_{\alpha i} \sim 10^{-2}$ and $m_{i} \sim 1 \mathrm{eV}$ for example, we find that the terms in the squre bracket of Eq. (29) are roughly at the level of $7.2 \times 10^{-9}$ for $M_{i} \sim 100 \mathrm{TeV}$ or $1.9 \times 10^{-5}$ for $M_{i} \sim 1 \mathrm{TeV}$. So the leading-order approximation made in Eq. (24) and its intriguing consequence obtained in Eq. (27) are actually safe enough for a natural seesaw mechanism with $M_{i} \gtrsim 100 \mathrm{TeV}$. The latter is essentially the defined regime of "heavy" sterile neutrinos in Sect. 2. If $M_{i} \sim 1 \mathrm{TeV}$ holds, however, the upper bound on $\left|\sum_{i=1}^{3} U_{e i} U_{\mu i}^{*}\right|$ achieved in Eq. (27) will suffer a large uncertainty induced by the nextto-leading-order contribution of $G_{\gamma}\left(M_{i}^{2} / M_{W}^{2}\right)$, but the upper limits on $\left|\sum_{i=1}^{3} U_{e i} U_{\tau i}^{*}\right|$ and $\left|\sum_{i=1}^{3} U_{\mu i} U_{\tau i}^{*}\right|$ remain valid.

The future precision measurements at low energies will allow us to test unitarity of the $3 \times 3$ PMNS matrix $U$ to a much better degree of accuracy. In this connection it makes sense to look at the geometry of a given unitarity polygon as compared with the geometry of its three sub-triangles $\triangle_{\alpha \beta}^{i j}$ formed by two vectors $U_{\alpha i} U_{\beta i}^{*}$ and $U_{\alpha j} U_{\beta j}^{*}$ (for $i \neq j$ ) in the complex plane, as illustrated by Fig. 5. The inner intersection angle between these two vectors can therefore be defined as

$$
\begin{aligned}
& \phi_{\gamma 1} \equiv \arg \left(-\frac{U_{\alpha 2} U_{\beta 2}^{*}}{U_{\alpha 3} U_{\beta 3}^{*}}\right), \quad \phi_{\gamma 2} \equiv \arg \left(-\frac{U_{\alpha 3} U_{\beta 3}^{*}}{U_{\alpha 1} U_{\beta 1}^{*}}\right), \\
& \phi_{\gamma 3} \equiv \arg \left(-\frac{U_{\alpha 1} U_{\beta 1}^{*}}{U_{\alpha 2} U_{\beta 2}^{*}}\right),
\end{aligned}
$$

where $(\alpha, \beta, \gamma)=(e, \mu, \tau),(\mu, \tau, e)$ or $(\tau, e, \mu)$. It is obvious that the sum

$\phi_{\gamma 1}+\phi_{\gamma 2}+\phi_{\gamma 3}=\pi$

holds by definition, even though $U_{\alpha 1} U_{\beta 1}^{*}+U_{\alpha 2} U_{\beta 2}^{*}+$ $U_{\alpha 3} U_{\beta 3}^{*} \neq 0$. This point is sometimes overlooked in the quark sector when discussing the CKM unitarity test.

One may establish a direct link between the areas of those sub-triangles $\triangle_{\alpha \beta}^{i j}$, denoted as $S_{\alpha \beta}^{i j}$, and the Jarlskog-like invariants which are defined in Eq. (4) and satisfy the relation 


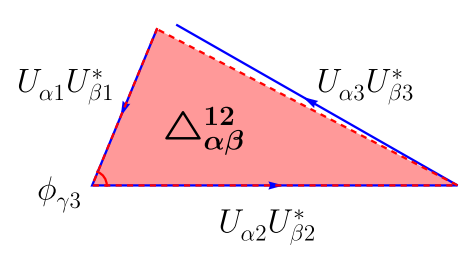

(a)

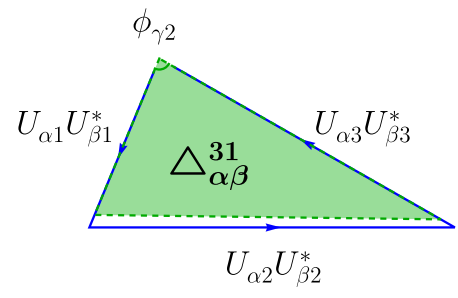

(b)

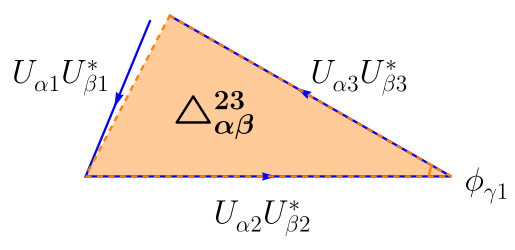

(c)

Fig. 5 Three sub-triangles $\triangle_{\alpha \beta}^{i j}$ of a given unitarity polygon with definite $\alpha$ and $\beta$ flavor indices, formed by two vectors $U_{\alpha i} U_{\beta i}^{*}$ and $U_{\alpha j} U_{\beta j}^{*}$ (for $i \neq j$ ) in the complex plane

$\mathcal{J}_{\alpha \beta}^{i j}=\mathcal{J}_{\beta \alpha}^{j i}=-\mathcal{J}_{\alpha \beta}^{j i}=-\mathcal{J}_{\beta \alpha}^{i j}$. Namely, we have

$S_{\alpha \beta}^{i j}=\frac{1}{2}\left|\mathcal{J}_{\alpha \beta}^{i j}\right|$

where $(\alpha, \beta)=(e, \mu),(\mu, \tau)$ or $(\tau, e)$ and $(i, j)=(1,2)$, $(2,3)$ or $(3,1)$. If $R=0$ and $U$ is exactly unitary, all the possible Jarlskog-like invariants are identical in magnitude [41]. This will not be true anymore in the existence of activesterile neutrino mixing, implying that the areas of $\triangle_{\alpha \beta}^{i j}$ are not exactly equal. To illustrate this point in a more transparent way, we consider the $(3+3)$ active-sterile neutrino mixing scenario and write out the explicit expressions of $U$ and $R$ in an Euler-like parametrization of the $6 \times 6$ unitary matrix $\mathcal{U}$, as outlined in Appendix C. As a result, we find

$\left|S_{e \mu}^{12}-S_{e \mu}^{23}\right|=\frac{1}{2}\left|s_{12} c_{13}\left(c_{12} c_{23} \operatorname{Im} X_{1}-s_{12} s_{13} s_{23} \operatorname{Im} X_{2}\right)\right|$,

$\left|S_{e \mu}^{12}-S_{e \mu}^{31}\right|=\frac{1}{2}\left|c_{12} c_{13}\left(s_{12} c_{23} \operatorname{Im} X_{1}+c_{12} s_{13} s_{23} \operatorname{Im} X_{2}\right)\right|$,

$\left|S_{e \mu}^{23}-S_{e \mu}^{31}\right|=\frac{1}{2}\left|c_{13} s_{13} s_{23} \operatorname{Im} X_{2}\right|$;

and

$\left|S_{\tau e}^{12}-S_{\tau e}^{23}\right|=\frac{1}{2}\left|s_{12} c_{13}\left(c_{12} s_{23} \operatorname{Im} Y_{1}+s_{12} s_{13} c_{23} \operatorname{Im} Y_{2}\right)\right|$,

$\left|S_{\tau e}^{12}-S_{\tau e}^{31}\right|=\frac{1}{2}\left|c_{12} c_{13}\left(s_{12} s_{23} \operatorname{Im} Y_{1}-c_{12} s_{13} c_{23} \operatorname{Im} Y_{2}\right)\right|$,

$\left|S_{\tau e}^{23}-S_{\tau e}^{31}\right|=\frac{1}{2}\left|c_{13} s_{13} c_{23} \operatorname{Im} Y_{2}\right|$;

as well as

$$
\begin{aligned}
\left|S_{\mu \tau}^{12}-S_{\mu \tau}^{23}\right|= & \frac{1}{2} \mid\left(c_{12}^{2}-s_{12}^{2} s_{13}^{2}\right) c_{23} s_{23} \operatorname{Im} Z_{1} \\
& +c_{12} s_{12} s_{13}\left(c_{23}^{2} \operatorname{Im} Z_{2}-s_{23}^{2} \operatorname{Im} Z_{3}\right) \mid \\
\left|S_{\mu \tau}^{12}-S_{\mu \tau}^{31}\right|= & \frac{1}{2} \mid\left(c_{12}^{2} s_{13}^{2}-s_{12}^{2}\right) c_{23} s_{23} \operatorname{Im} Z_{1} \\
& +c_{12} s_{12} s_{13}\left(c_{23}^{2} \operatorname{Im} Z_{2}-s_{23}^{2} \operatorname{Im} Z_{3}\right) \mid, \\
\left|S_{\mu \tau}^{23}-S_{\mu \tau}^{31}\right|= & \frac{1}{2}\left|c_{13}^{2} c_{23} s_{23} \operatorname{Im} Z_{1}\right|,
\end{aligned}
$$

where $c_{i j} \equiv \cos \theta_{i j}, s_{i j} \equiv \sin \theta_{i j}$ (for $i j=12,13,23$ ), and $X_{i}, Y_{i}$ and $Z_{i}$ (for $i=1,2,3$ ) are defined in
Eq. (C9) of Appendix $\mathrm{C}$ and depend on the active-sterile neutrino mixing angles and $\mathrm{CP}$-violating phases. Equations (C6)-(C8) tell us that all the nine Jarlskog-like invariants $\mathcal{J}_{\alpha \beta}^{i j}$ will be reduced to the unique Jarlskog invariant $\mathcal{J}_{0}=\sin 2 \theta_{12} \sin 2 \theta_{13} \cos \theta_{13} \sin 2 \theta_{23} \sin \delta / 8$ in the standard parametrization of $U$ if $U$ is exactly unitary, and in this case triangles $\triangle_{\alpha \beta}^{12}, \triangle_{\alpha \beta}^{23}$ and $\triangle_{\alpha \beta}^{31}$ are equivalent to one another and their areas are all equal to $\left|\mathcal{J}_{0}\right| / 2$. Since $X_{i}, Y_{i}$ and $Z_{i}$ (for $i=1,2,3)$ are all of $\mathcal{O}\left(\sin ^{2} \theta_{i j}\right)$ with $\theta_{i j} \lesssim \mathcal{O}(0.1)$ being the small active-sterile neutrino mixing angles (for $i=1,2,3$ and $j=4,5,6)$, the areas of three sub-triangles of a given unitarity hexagon are expected to be different from one another at most at the level of one percent or much smaller. Such an observation is certainly true for a generic unitarity polygon, and that is why the latter can be reduced to an effective unitarity triangle with an effective apex as illustrated by Fig. 1.

Here it is worth mentioning that the areas of triangles $\triangle_{\alpha \beta}^{12}$, $\triangle_{\alpha \beta}^{23}$ and $\triangle_{\alpha \beta}^{31}$ can in principle be measured in some longbaseline neutrino oscillation experiments. To see this point, let us assume all the sterile particles in our $(3+n)$ activesterile neutrino mixing scheme to be kinematically forbidden and write out the possibilities of active $v_{\alpha} \rightarrow v_{\beta}$ oscillations in vacuum [27]:

$$
\begin{aligned}
& P\left(v_{\alpha} \rightarrow v_{\beta}\right) \\
& =\frac{\sum_{i=1}^{3}\left|U_{\alpha i}\right|^{2}\left|U_{\beta i}\right|^{2}+2 \sum_{i<j} \operatorname{Re}\left(U_{\alpha i} U_{\beta j} U_{\alpha j}^{*} U_{\beta i}^{*}\right) \cos \Delta_{i j}-2 \sum_{i<j} \mathcal{J}_{\alpha \beta}^{i j} \sin \Delta_{i j}}{\left(U U^{\dagger}\right)_{\alpha \alpha}\left(U U^{\dagger}\right)_{\beta \beta}},
\end{aligned}
$$

where $\Delta_{i j} \equiv\left(m_{i}^{2}-m_{j}^{2}\right) L /(2 E)$. The possibilities of $\bar{v}_{\alpha} \rightarrow \bar{v}_{\beta}$ oscillations in vacuum can be easily achieved from Eq. (36) by making the replacement $U \rightarrow U^{*}$. Then the CPviolating asymmetries between $v_{\alpha} \rightarrow v_{\beta}$ and $\bar{v}_{\alpha} \rightarrow \bar{v}_{\beta}$ oscillations are directly determined by the relevant Jarlskoglike invariants as follows:

$\mathcal{A}_{\alpha \beta} \equiv P\left(v_{\alpha} \rightarrow v_{\beta}\right)-P\left(\bar{v}_{\alpha} \rightarrow \bar{v}_{\beta}\right)$ 


$$
\begin{aligned}
& 4 \sum_{i<j} \mathcal{J}_{\alpha \beta}^{i j} \sin \Delta_{i j} \\
=- & \frac{\left(U U^{\dagger}\right)_{\alpha \alpha}\left(U U^{\dagger}\right)_{\beta \beta}}{\simeq-4 \sum_{i<j}\left[1+\left(R R^{\dagger}\right)_{\alpha \alpha}+\left(R R^{\dagger}\right)_{\beta \beta}\right] \mathcal{J}_{\alpha \beta}^{i j} \sin \Delta_{i j},}
\end{aligned}
$$

in which the small active-sterile neutrino mixing effects hidden in $R$ have been taken into account. Note that the $\left(R R^{\dagger}\right)_{\alpha \alpha}+\left(R R^{\dagger}\right)_{\beta \beta}$ term will cancel the $\mathcal{X}^{\prime}, \mathcal{Y}^{\prime}$ or $\mathcal{Z}^{\prime}$ term in the expressions of $\mathcal{J}_{\alpha \beta}^{i j}$ as shown in Eqs. (C6)-(C10), but the combinations $\left[1+\left(R R^{\dagger}\right)_{\alpha \alpha}+\left(R R^{\dagger}\right)_{\beta \beta}\right] \mathcal{J}_{\alpha \beta}^{i j}$ are still different from $\mathcal{J}_{0}$ because of $R \neq 0$. Of course, one has to take into account the terrestrial matter effects on $v_{\alpha} \rightarrow v_{\beta}$ and $\bar{v}_{\alpha} \rightarrow \bar{v}_{\beta}$ oscillations in a realistic long-baseline experiment $[27,47]$. Moreover, the detector efficiencies and the associated systematics should be taken into consideration to probe the tiny $R$-induced corrections to $\mathcal{A}_{\alpha \beta}$. All such and other possible uncertainties are much larger than the strength of $R$ induced CP violation in current experimental environments, and hence it will be extremely difficult (if not impossible) to establish a convincing signal of this kind of new physics even in the foreseeable future.

Finally, let us make some brief comments on the situation that the sterile neutrinos are light enough such that $M_{i} \ll$ $M_{W}$ holds (for $i=1,2, \ldots, n$ ) [48]. In this case, the result of $\xi\left(\beta^{-} \rightarrow \alpha^{-}+\gamma\right)$ obtained in Eq. (21) can be simplified to

$\xi\left(\beta^{-} \rightarrow \alpha^{-}+\gamma\right) \simeq \frac{3 \alpha_{\mathrm{em}}}{32 \pi M_{W}^{4}}\left|\langle M\rangle_{\alpha \beta}^{2}\right|^{2}$,

where the effective neutrino mass $\langle M\rangle_{\alpha \beta}$ are defined as

$\langle M\rangle_{\alpha \beta}^{2}=\sum_{i=1}^{3} m_{i}^{2} U_{\alpha i} U_{\beta i}^{*}+\sum_{i=1}^{n} M_{i}^{2} R_{\alpha i} R_{\beta i}^{*}$.

It is obvious that $\langle M\rangle_{\alpha \beta}$ is insensitive to the Majorana phases hidden in $U$ and $R$, and thus it is closely related to lepton flavor violation in the charged-lepton sector. Taking account of current experimental bounds on $\xi\left(\beta^{-} \rightarrow \alpha^{-}+\gamma\right)$ given in Eq. (26), we immediately arrive at

$$
\begin{aligned}
& \left|\langle M\rangle_{e \mu}\right|<0.53 \mathrm{GeV}, \quad\left|\langle M\rangle_{e \tau}\right|<13.73 \mathrm{GeV}, \\
& \left|\langle M\rangle_{\mu \tau}\right|<14.84 \mathrm{GeV} .
\end{aligned}
$$

Such upper limits remain too large to really probe the magnitudes of $m_{i}$ and $M_{i}$ in $\langle M\rangle_{\alpha \beta}$.

\section{Summary}

We are entering the era of precision measurements of both flavor oscillations of massive neutrinos and lepton flavor violation in the charged-lepton sector. A burning issue is to test unitarity of the $3 \times 3$ PMNS matrix $U$ so as to probe or constrain possible new but sterile degrees of freedom which may slightly mix with three active neutrino species. The most popular example of this kind is the heavy Majorana neutrinos in the canonical seesaw mechanism, although much lighter sterile neutrinos are also taken into account in some low-scale seesaw models or purely from a phenomenological point of view. In this connection the radiative decays of charged leptons in the form of $\beta^{-} \rightarrow \alpha^{-}+\gamma$, which may take place via both active and sterile neutrinos in the one-loop, are expected to be an ideal tool to examine the departure of a unitarity polygon from the standard unitarity triangle of $U$ in the $(3+n)$ active-sterile neutrino mixing scheme.

That is why we have calculated the rates of radiative $\beta^{-} \rightarrow \alpha^{-}+\gamma$ decays in the unitary gauge, and confirmed the results obtained previously in Refs. [30,31]. ${ }^{3}$ We have made it clear that constraints on the unitarity of $U$ extracted from $\beta^{-} \rightarrow \alpha^{-}+\gamma$ decays in the minimal unitarity violation scheme differ from those obtained in the canonical seesaw mechanism with $n$ heavy Majorana neutrinos by a factor $5 / 3$. In such a natural seesaw case we have demonstrated that the rates of $\beta^{-} \rightarrow \alpha^{-}+\gamma$ can be used to cleanly and strongly constrain the effective apex of a unitarity polygon as shown in Fig. 1, and discussed its geometry as compared with the geometry of its three sub-triangles $\triangle_{\alpha \beta}^{i j}$ formed by two vectors $U_{\alpha i} U_{\beta i}^{*}$ and $U_{\alpha j} U_{\beta j}^{*}$ (for $i \neq j$ ) in the complex plane. It is found that the areas of such sub-triangles can be described in terms of the Jarlskog-like invariants of CP violation $\mathcal{J}_{\alpha \beta}^{i j}$, and their small differences may serve as a signal of slight unitarity violation of the $3 \times 3$ PMNS matrix $U$. These observations should be useful to test the unitarity of $U$ when more accurate experimental data are available in the foreseeable future, and they can certainly be extended to those simplified seesaw cases (e.g., the minimal seesaw scenarios [51]) with fewer free parameters.

Acknowledgements We would like to thank Enrique FernandezMartinez, Zhi-cheng Liu and Shun Zhou for very useful discussions. This work was supported in part by the National Natural Science Foundation of China under grant No. 11775231 and grant No. 11835013.

Data Availability Statement This manuscript has no associated data or the data will not be deposited. [Authors' comment:Data sharing not applicable to this article as no datasets were generated or analysed during the current study.]

Open Access This article is licensed under a Creative Commons Attribution 4.0 International License, which permits use, sharing, adaptation, distribution and reproduction in any medium or format, as long as you give appropriate credit to the original author(s) and the source, provide a link to the Creative Commons licence, and indicate if changes were made. The images or other third party material in this article

\footnotetext{
3 Throughout this work we have focused on the non-supersymmetric active-sterile neutrino mixing scheme. We refer the reader to Refs. [49, 50] for charged lepton flavor violation in the supersymmetric seesaw scenarios.
} 
are included in the article's Creative Commons licence, unless indicated otherwise in a credit line to the material. If material is not included in the article's Creative Commons licence and your intended use is not permitted by statutory regulation or exceeds the permitted use, you will need to obtain permission directly from the copyright holder. To view a copy of this licence, visit http://creativecomm ons.org/licenses/by/4.0/.

Funded by SCOAP ${ }^{3}$.

\section{A Dimensional regularization}

In $D \equiv 4-\varepsilon$ dimensions, the Dirac matrices satisfy

$$
\left\{\gamma^{\mu}, \gamma^{\nu}\right\}=2 g^{\mu \nu}
$$

and the Minkowski metric tensor $g^{\mu \nu}$ satisfies

$$
\begin{aligned}
g^{\mu \nu} & =g^{\nu \mu}, \\
g^{\mu \rho} g_{\rho}^{v} & =g^{\mu \nu}, \\
g^{\mu \nu} g_{\mu \nu} & =D .
\end{aligned}
$$

Given Eqs. (A1) and (A2), one may derive

$$
\begin{aligned}
\gamma^{\mu} \gamma_{\mu} & =D, \\
\gamma^{\mu} \gamma^{\nu} \gamma_{\mu} & =-(D-2) \gamma^{\nu}, \\
\gamma^{\mu} \gamma^{\nu} \gamma^{\rho} \gamma_{\mu} & =4 g^{\nu \rho}-(4-D) \gamma^{\nu} \gamma^{\rho}, \\
\gamma^{\mu} \gamma^{\nu} \gamma^{\rho} \gamma^{\sigma} \gamma_{\mu} & =-2 \gamma^{\sigma} \gamma^{\rho} \gamma^{\nu}+(4-D) \gamma^{\nu} \gamma^{\rho} \gamma^{\sigma} .
\end{aligned}
$$

Traces of the Dirac matrices which do not contain $\gamma_{5}$ keep unchanged as compared with those in the 4-dimensional case; that is,

$$
\operatorname{Tr}(\mathbf{1})=4,
$$

$\operatorname{Tr}\left(\right.$ odd number of $\left.\gamma^{\prime} \mathrm{s}\right)=0$,

$$
\begin{aligned}
\operatorname{Tr}\left(\gamma^{\mu} \gamma^{\nu}\right) & =4 g^{\mu \nu}, \\
\operatorname{Tr}\left(\gamma^{\mu} \gamma^{\nu} \gamma^{\rho} \gamma^{\sigma}\right) & =4\left(g^{\mu \nu} g^{\rho \sigma}-g^{\mu \rho} g^{\nu \sigma}+g^{\mu \sigma} g^{\nu \rho}\right),
\end{aligned}
$$

where the first relation is just a convention. In $D$ dimensions, the issue of $\gamma_{5}$ is quite subtle, but we can simply use the "naive dimensional regularization" [52], namely

$$
\left\{\gamma^{\mu}, \gamma_{5}\right\}=0
$$

Although the above relation leads to obvious algebraic inconsistencies [53-56], there occurs no trouble concerning the $\gamma_{5}$ matrix in the present work [52].

In $D$ dimensions, we also need to deal with the dimensions of all the fields and couplings in the Lagrangian. To keep the action dimensionless, the Lagrangian density should have mass dimension $D$. For simplicity, we take the QED theory as an example, where the Lagrangian is given by

$$
\begin{aligned}
\mathcal{L}_{\mathrm{QED}}= & -\frac{1}{4}\left(\partial_{\mu} A_{v}-\partial_{\nu} A_{\mu}\right)^{2}+\bar{\psi}\left(\mathrm{i} \gamma^{\mu} \partial_{\mu}-m\right) \psi \\
& -e \bar{\psi} \gamma^{\mu} \psi A_{\mu},
\end{aligned}
$$

with $A_{\mu}$ and $\psi$ being the massless gauge field and the massive fermion field, respectively. The kinetic and mass terms in Eq. (A6) imply the mass dimensions

$$
[m]=1, \quad\left[A_{\mu}\right]=\frac{D-2}{2}, \quad[\psi]=\frac{D-1}{2} .
$$

Then with the help of Eq. (A7) and the interaction term in Eq. (A6), one can achieve $[e]=(4-D) / 2$, where the coupling $e$ is no longer dimensionless and has a non-integer mass dimension. To keep $e$ dimensionless, it is conventional to make the replacement

$e \rightarrow \mu^{\frac{4-D}{2}} e$

where $\mu$ is an arbitrary mass-dimension-one parameter. It is also true for the coupling $g$ in Eq. (2) that the replacement $g \rightarrow \mu^{(4-D) / 2} g$ is made for the purpose of keeping $g$ dimensionless in $D$ dimensions. Thus in $D$ dimensions, all the Feynman rules for those vertices involving the couplings $e$ and $g$ should take the replacements

$e \rightarrow \mu^{\frac{4-D}{2}} e, \quad g \rightarrow \mu^{\frac{4-D}{2}} g$

\section{B The Passarino-Veltman functions}

Taking account of the on-shell conditions $p^{2}=m_{\mu}^{2}, q^{2}=0$ and $p \cdot q=\left(m_{\mu}^{2}-m_{e}^{2}\right) / 2$, the physical polarizations for the external photon $\epsilon(q) \cdot q=0$ and the Passarino-Veltman integrals, we may rewrite the three one-loop Feynman-diagram amplitudes in Eq. (5) as follows:

$$
\begin{aligned}
\mathrm{i} \mathcal{M}_{\mathrm{a}}= & \frac{\mathrm{i} e g^{2}}{2(4 \pi)^{2}} \epsilon_{\rho}^{*}(q) \bar{u}(p-q) \\
& \times \frac{m_{e}\left(m_{e} P_{\mathrm{R}}+m_{\mu} P_{\mathrm{L}}\right)}{m_{\mu}^{2}-m_{e}^{2}} \gamma^{\rho} u(p) \sum_{i=1}^{3+n} \mathcal{U}_{e i} \mathcal{U}_{\mu i}^{*} \mu^{\frac{1}{2} \varepsilon} \\
& \times\left\{(2-D) B_{0}(p-q)+(1-D) B_{1}(p-q)\right. \\
& +\frac{1}{M_{W}^{2}}\left[(D-2) B_{00}(p-q)\right. \\
& \left.\left.-m_{e}^{2} B_{11}(p-q)+A_{0}\left(\lambda_{i}\right)\right]\right\}, \\
\mathrm{i} \mathcal{M}_{\mathrm{b}}= & -\frac{\mathrm{i} e g^{2}}{2(4 \pi)^{2}} \epsilon_{\rho}^{*}(q) \bar{u}(p-q) \\
& \times \frac{m_{\mu}\left(m_{e} P_{\mathrm{L}}+m_{\mu} P_{\mathrm{R}}\right)}{m_{\mu}^{2}-m_{e}^{2}} \gamma^{\rho} u(p) \sum_{i=1}^{3+n} \mathcal{U}_{e i} \mathcal{U}_{\mu i}^{*} \mu^{\frac{1}{2} \varepsilon} \\
& \times\left\{(2-D) B_{0}(p)+(1-D) B_{1}(p)\right. \\
& \left.+\frac{1}{M_{W}^{2}}\left[(D-2) B_{00}(p)-m_{\mu}^{2} B_{11}(p)+A_{0}\left(\lambda_{i}\right)\right]\right\},
\end{aligned}
$$


and

$\mathrm{i} \mathcal{M}_{\mathrm{c}}=-\frac{\mathrm{i} e g^{2}}{2(4 \pi)^{2}} \epsilon_{\rho}^{*} \sum_{i=1}^{3+n} \mathcal{U}_{e i} \mathcal{U}_{\mu i}^{*} \bar{u}(p-q) \mu^{\frac{1}{2} \varepsilon} \mathcal{M}^{\rho}(p) u(p)$

with

$$
\begin{aligned}
\mathcal{M}^{\rho}= & 2 m_{\mu} p^{\rho} P_{\mathrm{R}}\left\{C_{0}-C_{1}+C_{2}-(D-1) C_{12}\right. \\
& +\frac{1}{M_{W}^{2}}\left[B_{1}(p)+B_{11}(p)+(D-2) C_{00}+D C_{001}\right. \\
& \left.\left.-m_{e}^{2} C_{122}\right]\right\}+2 m_{e} p^{\rho} P_{\mathrm{L}}\left\{2 C_{0}+C_{1}+D C_{2}\right. \\
& +(D-1)\left(C_{12}+C_{22}\right)+\frac{1}{M_{W}^{2}}\left[-B_{1}(p)-2 C_{00}\right. \\
& +m_{e}^{2} C_{22}-D C_{001}+4 C_{002}+2 m_{e}^{2} C_{222} \\
& \left.\left.+\left(2 m_{e}^{2}-m_{\mu}^{2}\right) C_{122}\right]\right\}-m_{e} m_{\mu} \gamma^{\rho} P_{\mathrm{R}}\left\{3\left(C_{0}+C_{2}\right)\right. \\
& +\frac{1}{M_{W}^{2}}\left[(D-4) C_{00}+m_{e}^{2}\left(C_{22}+C_{222}\right)\right. \\
& \left.\left.+(D+2) C_{002}-\left(m_{\mu}^{2}-m_{e}^{2}\right) C_{122}\right]\right\} \\
& -\gamma^{\rho} P_{\mathrm{L}}\left\{\left(m_{e}^{2}+2 m_{\mu}^{2}\right) C_{0}+\left(m_{\mu}^{2}-m_{e}^{2}\right) C_{1}\right. \\
& +\left(2 m_{\mu}^{2}-m_{e}^{2}\right) C_{2}-2(D-1) C_{00} \\
& +\frac{1}{M_{W}^{2}}\left[\left(m_{\mu}^{2}-m_{e}^{2}\right)\left[2 B_{1}(p)-D C_{001}\right]\right. \\
& +2(D-1) B_{00}(p)+2 m_{\mu}^{2} B_{11}(p) \\
& +\left[(D-2) m_{e}^{2}-2 m_{\mu}^{2}\right] C_{00}+\left(m_{\mu}^{2}-m_{e}^{2}\right)^{2}\left(C_{12}\right. \\
& \left.+C_{112}\right)-m_{e}^{2}\left(m_{\mu}^{2}-2 m_{e}^{2}\right) C_{22} \\
& -\left[(D+2) m_{\mu}^{2}-4(D+1) m_{e}^{2}\right] C_{002} \\
& +\left(m_{\mu}^{2}-5 m_{e}^{2}\right)\left(m_{\mu}^{2}-m_{e}^{2}\right) C_{122} \\
& \left.\left.-m_{e}^{2}\left(m_{\mu}^{2}-4 m_{e}^{2}\right) C_{222}\right]\right\} \\
& \left(D-D^{2}\right. \\
&
\end{aligned}
$$

where $A_{0}\left(\lambda_{i}\right), B_{0}\left(p^{\prime}\right) \equiv B_{0}\left(p^{\prime}, M_{W}, \lambda_{i}\right)$ (for $p^{\prime}=p$ or $p-q)$ and $C_{0} \equiv C_{0}\left(q, q-p, M_{W}, M_{W}, \lambda_{i}\right)$ are the Passarino-Veltman scalar integrals whose generic forms are defined as [57-59]

$$
\begin{aligned}
& A_{0}(\lambda)=\frac{(2 \pi \mu)^{4-D}}{\mathrm{i} \pi^{2}} \int \mathrm{d}^{D} k \frac{1}{k^{2}-\lambda^{2}}, \\
& B_{0}\left(p, \lambda_{1}, \lambda_{2}\right) \\
&= \frac{(2 \pi \mu)^{4-D}}{\mathrm{i} \pi^{2}} \int \mathrm{d}^{D} k \frac{1}{\left(k^{2}-\lambda_{1}^{2}\right)\left[(k+p)^{2}-\lambda_{2}^{2}\right]}, \\
& C_{0}\left(p_{1}, p_{2}, \lambda_{1}, \lambda_{2}, \lambda_{3}\right) \\
&=\frac{(2 \pi \mu)^{4-D}}{\mathrm{i} \pi^{2}} \int \mathrm{d}^{D} k \\
& \quad \times \frac{1}{\left(k^{2}-\lambda_{1}^{2}\right)\left[\left(k+p_{1}\right)^{2}-\lambda_{2}^{2}\right]\left[\left(k+p_{2}\right)^{2}-\lambda_{3}^{2}\right]},
\end{aligned}
$$

and $B_{x}\left(p^{\prime}\right)$ (for $\left.x=1,00,11\right)$ and $C_{y}$ (for $y=$ $1,2,00,11,12,22,001,002,112,122,222)$ are the Passarino-
Veltman coefficient functions for the tension integral decompositions which contain the same parameters as $B_{0}\left(p^{\prime}\right)$ and $C_{0}$, respectively. Up to the order of $\mathcal{O}\left(m_{\alpha}^{2} / M_{W}^{2}\right)$ for $\alpha=e$ or $\mu$, the Passarino-Veltman functions appearing in Eqs. (B1)(B4) are explicitly given by $[60,61]$

$A_{0}\left(\lambda_{i}\right)=\lambda_{i}^{2}\left(\Delta-\ln \frac{M_{W}^{2}}{\mu^{2}}-\ln x_{i}+1\right) ;$

and

$$
\begin{aligned}
& B_{0}\left(p^{\prime}\right) \simeq 1+\Delta-\frac{x_{i}}{x_{i}-1} \ln x_{i} \\
&+\frac{x_{i}^{2}-1-2 x_{i} \ln x_{i}}{2\left(x_{i}-1\right)^{3}} \cdot \frac{m_{\alpha}^{2}}{M_{W}^{2}}-\ln \frac{M_{W}^{2}}{\mu^{2}} \\
& B_{1}\left(p^{\prime}\right) \simeq-\frac{1}{2} \Delta+\frac{-x_{i}^{2}+4 x_{i}-3+2 x_{i}\left(x_{i}-2\right) \ln x_{i}}{4\left(x_{i}-1\right)^{2}} \\
&-\frac{x_{i}^{3}-6 x_{i}^{2}+3 x_{i}+2+6 x_{i} \ln x_{i}}{6\left(x_{i}-1\right)^{4}} \cdot \frac{m_{\alpha}^{2}}{M_{W}^{2}} \\
& B_{00}\left(p^{\prime}\right) \simeq \frac{3 M_{W}^{2}\left(x_{i}+1\right)-m_{\alpha}^{2}}{12} \Delta+\left[\frac{1}{12} \ln \frac{M_{W}^{2}}{\mu^{2}}\right. \\
&\left.+\frac{-5 x_{i}^{3}+27 x_{i}^{2}-27 x_{i}+5+6 x_{i}^{2}\left(x_{i}-3\right) \ln x_{i}}{72\left(x_{i}-1\right)^{3}}\right] m_{\alpha}^{2} \\
&+\frac{3\left(x_{i}^{2}-1\right)-2 x_{i}^{2} \ln x_{i}}{8\left(x_{i}-1\right)} M_{W}^{2} \\
&-\frac{1}{4} M_{W}^{2}\left(x_{i}+1\right) \ln \frac{M_{W}^{2}}{\mu^{2}}, \\
& \frac{1}{M_{W}^{2}}-\frac{1}{3} \ln \frac{M_{W}^{2}}{\mu^{2}} ; \\
& B_{11}\left(p^{\prime}\right) \simeq \frac{2 x_{i}^{3}-9 x_{i}^{2}+18 x_{i}-11-6 x_{i}\left(x_{i}^{2}-3 x_{i}+3\right) \ln x_{i}}{18\left(x_{i}-1\right)^{3}} \\
&+\frac{x_{i}^{4}-6 x_{i}^{3}+18 x_{i}^{2}-10 x_{i}-3-12 x_{i} \ln x_{i}}{5} \\
& \hline
\end{aligned}
$$

and

$$
\begin{aligned}
C_{0} \simeq & \frac{x_{i}-1-x_{i} \ln x_{i}}{M_{W}^{2}\left(x_{i}-1\right)^{2}}+\left(m_{e}^{2}+m_{\mu}^{2}\right) \\
& \times \frac{5 x_{i}^{2}-4 x_{i}-1-2 x_{i}\left(x_{i}+2\right) \ln x_{i}}{4 M_{W}^{4}\left(x_{i}-1\right)^{2}}, \\
C_{1} \simeq & -\frac{3 x_{i}^{2}-4 x_{i}+1-2 x_{i}^{2} \ln x_{i}}{4 M_{W}^{2}\left(x_{i}-1\right)^{3}}+\left(m_{e}^{2}+2 m_{\mu}^{2}\right) \\
& \times \frac{-17 x_{i}^{3}+9 x_{i}^{2}+9 x_{i}-1+6 x_{i}^{2}\left(x_{i}+3\right) \ln x_{i}}{36 M_{W}^{4}\left(x_{i}-1\right)^{5}}, \\
C_{2} \simeq & \frac{x_{i}^{2}-1-2 x_{i} \ln x_{i}}{2 M_{W}^{2}\left(x_{i}-1\right)^{3}+\left(m_{e}^{2}+m_{\mu}^{2}\right)} \\
& \times \frac{x_{i}^{3}+9 x_{i}^{2}-9 x_{i}-1-6 x_{i}\left(x_{i}+1\right) \ln x_{i}}{6 M_{W}^{4}\left(x_{i}-1\right)^{5}}, \\
C_{00} \simeq & \frac{1}{4} \Delta+\frac{3 x_{i}^{2}-4 x_{i}+1-2 x_{i}^{2} \ln x_{i}}{8\left(x_{i}-1\right)^{2}}
\end{aligned}
$$




$$
\begin{aligned}
& +\left(m_{e}^{2}+m_{\mu}^{2}\right) \frac{2 x_{i}^{3}+3 x_{i}^{2}-6 x_{i}+1-6 x_{i}^{2} \ln x_{i}}{24 M_{W}^{2}\left(x_{i}-1\right)^{4}} \\
& -\frac{1}{4} \ln \frac{M_{W}^{2}}{\mu^{2}}, \\
& C_{12} \simeq-\frac{2 x_{i}^{3}+3 x_{i}^{2}-6 x_{i}+1-6 x_{i}^{2} \ln x_{i}}{12 M_{W}^{2}\left(x_{i}-1\right)^{4}}-\left(m_{e}^{2}+2 m_{\mu}^{2}\right) \\
& \times \frac{3 x_{i}^{4}+44 x_{i}^{3}-36 x_{i}^{2}-12 x_{i}+1-12 x_{i}^{2}\left(2 x_{i}+3\right) \ln x_{i}}{72 M_{W}^{4}\left(x_{i}-1\right)^{6}}, \\
& C_{22} \simeq-\frac{x_{i}^{3}-6 x_{i}^{2}+3 x_{i}+2+6 x_{i} \ln x_{i}}{6 M_{W}^{2}\left(x_{i}-1\right)^{4}}-\left(m_{e}^{2}+m_{\mu}^{2}\right) \\
& \times \frac{x_{i}^{4}-12 x_{i}^{3}-36 x_{i}^{2}+44 x_{i}+3+12 x_{i}\left(3 x_{i}+2\right) \ln x_{i}}{24 M_{W}^{4}\left(x_{i}-1\right)^{6}}, \\
& C_{001} \simeq-\frac{1}{12} \Delta-\frac{11 x_{i}^{3}-18 x_{i}^{2}+9 x_{i}-2-6 x_{i}^{3} \ln x_{i}}{72\left(x_{i}-1\right)^{3}} \\
& -\left(m_{e}^{2}+2 m_{\mu}^{2}\right) \\
& \times \frac{3 x_{i}^{4}+10 x_{i}^{3}-18 x_{i}^{2}+6 x_{i}-1-12 x_{i}^{3} \ln x_{i}}{144 M_{W}^{2}\left(x_{i}-1\right)^{5}} \\
& +\frac{1}{12} \ln \frac{M_{W}^{2}}{\mu^{2}}, \\
& C_{002} \simeq-\frac{1}{12} \Delta-\frac{5 x_{i}^{3}-27 x_{i}^{2}+27 x_{i}-5-6 x_{i}^{2}\left(x_{i}-3\right) \ln x_{i}}{72\left(x_{i}-1\right)^{3}} \\
& -\left(m_{e}^{2}+m_{\mu}^{2}\right) \\
& \times \frac{x_{i}^{4}-8 x_{i}^{3}+8 x_{i}-1+12 x_{i}^{2} \ln x_{i}}{48 M_{W}^{2}\left(x_{i}-1\right)^{5}} \\
& +\frac{1}{12} \ln \frac{M_{W}^{2}}{\mu^{2}}, \\
& C_{112} \simeq \frac{3 x_{i}^{4}+10 x_{i}^{3}-18 x_{i}^{2}+6 x_{i}-1-12 x_{i}^{3} \ln x_{i}}{36 M_{W}^{2}\left(x_{i}-1\right)^{5}} \\
& +\left(m_{e}^{2}+3 m_{\mu}^{2}\right) \\
& \times \frac{6 x_{i}^{5}+125 x_{i}^{4}-80 x_{i}^{3}-60 x_{i}^{2}+10 x_{i}-1-60 x_{i}^{3}\left(x_{i}+2\right) \ln x_{i}}{360 M_{W}^{4}\left(x_{i}-1\right)^{7}}, \\
& C_{122} \simeq \frac{x_{i}^{4}-8 x_{i}^{3}+8 x_{i}-1+12 x_{i}^{2} \ln x_{i}}{24 M_{W}^{2}\left(x_{i}-1\right)^{5}}+\left(m_{e}^{2}+2 m_{\mu}^{2}\right) \\
& \times \frac{x_{i}^{5}-15 x_{i}^{4}-80 x_{i}^{3}+80 x_{i}^{2}+15 x_{i}-1+60 x_{i}^{2}\left(x_{i}+1\right) \ln x_{i}}{120 M_{W}^{4}\left(x_{i}-1\right)^{7}}, \\
& C_{222} \simeq \frac{x_{i}^{4}-6 x_{i}^{3}+18 x_{i}^{2}-10 x_{i}-3-12 x_{i} \ln x_{i}}{12 M_{W}^{2}\left(x_{i}-1\right)^{5}}+\left(m_{e}^{2}+m_{\mu}^{2}\right) \\
& \times \frac{x_{i}^{5}-10 x_{i}^{4}+60 x_{i}^{3}+80 x_{i}^{2}-125 x_{i}-6-60 x_{i}\left(2 x_{i}+1\right) \ln x_{i}}{60 M_{W}^{4}\left(x_{i}-1\right)^{7}}
\end{aligned}
$$

where $\alpha=e($ or $\mu)$ when $p^{\prime}=p-q$ (or $\left.p\right), x_{i}=\lambda_{i}^{2} / M_{W}^{2}$ and $\Delta \equiv 2 / \varepsilon-\gamma_{\mathrm{E}}+\ln (4 \pi)$ with $\gamma_{\mathrm{E}}$ being Euler's constant.

\section{The Jarlskog-like invariants}

In the $(3+3)$ active-sterile neutrino mixing scheme, the $6 \times 6$ unitary matrix $\mathcal{U}$ can be expressed as [28]

$\mathcal{U}=\left(\begin{array}{cc}I & 0 \\ 0 & U_{0}^{\prime}\end{array}\right)\left(\begin{array}{ll}A & R \\ S & B\end{array}\right)\left(\begin{array}{cc}U_{0} & 0 \\ 0 & I\end{array}\right)$,

where $I$ denotes the $3 \times 3$ identity matrix, $U_{0}$ and $U_{0}^{\prime}$ are unitary matrices responsible respectively for flavor mixing in the active sector and that in the sterile sector, and $A, B, R$ and $S$ are the $3 \times 3$ matrices describing the interplay between the two sectors. Then an Euler-like parametrization of $\mathcal{U}$ is

$$
\begin{aligned}
& \left(\begin{array}{cc}
U_{0} & 0 \\
0 & I
\end{array}\right)=O_{23} O_{13} O_{12}, \\
& \left(\begin{array}{cc}
I & 0 \\
0 & U_{0}^{\prime}
\end{array}\right)=O_{56} O_{46} O_{45}, \\
& \left(\begin{array}{cc}
A & R \\
S & B
\end{array}\right)=O_{36} O_{26} O_{16} O_{35} O_{25} O_{15} O_{34} O_{24} O_{14},
\end{aligned}
$$

where $O_{i j}$ (for $1 \leq i<j \leq 6$ ) are the two-dimensional $6 \times 6$ rotation matrices in the complex plane [27,28]. The elements of $O_{i j}$ satisfy $O_{i j}(i, i)=O_{i j}(j, j)=\cos \theta_{i j}$, $O_{i j}(i, j)=-O_{i j}^{*}(j, i)=\sin \theta_{i j} \exp \left(-\mathrm{i} \delta_{i j}\right), O_{i j}(n, n)=$ 1 (for $n \neq i, j$ ), and all the other elements are vanishing. So there are totally fifteen mixing angles $\theta_{i j}$ and fifteen $\mathrm{CP}$ violating phases $\delta_{i j}$ in the parametrization of $\mathcal{U}$. The PMNS matrix $U$ is actually a product of $A$ and $U_{0}$; namely, $U \equiv$ $A U_{0}$, where $A$ measures the departure of $U$ from $U_{0}$. The unitarity of $\mathcal{U}$ assures $U U^{\dagger}+R R^{\dagger}=A A^{\dagger}+R R^{\dagger}=I$, and the exact canonical seesaw formula is given by

$U D_{\nu} U^{T}+R D_{N} R^{T}=0$,

where $D_{v} \equiv \operatorname{Diag}\left\{m_{1}, m_{2}, m_{3}\right\}$ and $D_{N} \equiv \operatorname{Diag}\left\{M_{1}, M_{2}\right.$, $M_{3}$ \} are defined to be the diagonal active and sterile neutrino mass matrices, respectively. To calculate the Jarlskog-like invariants $\mathcal{J}_{\alpha \beta}^{i j}$ defined in Eq. (4), let us write out the expressions of $U_{0}$ and $A$ [2]:

$$
U_{0}=\left(\begin{array}{ccc}
c_{12} c_{13} & \hat{s}_{12}^{*} c_{13} & \hat{s}_{13}^{*} \\
-\hat{s}_{12} c_{23}-c_{12} \hat{s}_{13} \hat{s}_{23}^{*} & c_{12} c_{23}-\hat{s}_{12}^{*} \hat{s}_{13} \hat{s}_{23}^{*} & c_{13} \hat{s}_{23}^{*} \\
\hat{s}_{12} \hat{s}_{23}-c_{12} \hat{s}_{13} c_{23} & -c_{12} \hat{s}_{23}-\hat{s}_{12}^{*} \hat{s}_{13} c_{23} & c_{13} c_{23}
\end{array}\right),
$$

and 


$$
A=\left(\begin{array}{ccc}
c_{14} c_{15} c_{16} & 0 & 0 \\
-c_{14} c_{15} \hat{s}_{16} \hat{s}_{26}^{*}-c_{14} \hat{s}_{15} \hat{s}_{25}^{*} c_{26} & c_{24} c_{25} c_{26} & 0 \\
-\hat{s}_{14} \hat{s}_{24}^{*} c_{25} c_{26} & \\
-c_{14} c_{15} \hat{s}_{16} c_{22} \hat{s}_{36}^{*}+c_{14} \hat{s}_{15} \hat{s}_{25}^{*} \hat{s}_{26} \hat{s}_{36}^{*} & -c_{24} c_{25} \hat{s}_{26} \hat{s}_{36}^{*}-c_{24} \hat{s}_{25} \hat{s}_{35}^{*} c_{36} & \\
-c_{14} \hat{s}_{15} c_{25} \hat{s}_{35}^{*} c_{36}+\hat{s}_{14} \hat{s}_{24}^{*} c_{25} \hat{s}_{26} \hat{s}_{36}^{*} & -\hat{s}_{24} \hat{s}_{34}^{*} c_{35} c_{36} & \\
+\hat{s}_{14} \hat{s}_{24}^{*} \hat{s}_{25} \hat{s}_{35}^{*} c_{36}-\hat{s}_{14} c_{24} \hat{s}_{34}^{*} c_{35} c_{36} & c_{36}
\end{array}\right),
$$

where $\hat{s}_{i j} \equiv e^{\mathrm{i} \delta_{i j}} \sin \theta_{i j}$. Since the active-sterile neutrino mixing angles $\theta_{i j}$ (for $i=1,2,3$ and $j=4,5,6$ ) are expected to at most of $\mathcal{O}(0.1)$, one may make some reasonable analytical approximations to simplify the elements of $A$ up to $\mathcal{O}\left(10^{-2}\right)$. Then we obtain the results of nine Jarlskoglike invarants as follows:

$$
\begin{aligned}
\mathcal{J}_{e \mu}^{12} \simeq & \left(1-\mathcal{X}^{\prime}\right) \mathcal{J}_{0}+c_{12} s_{12} c_{13}^{3} c_{23} \operatorname{Im} X_{1}, \\
\mathcal{J}_{e \mu}^{23} \simeq & \left(1-\mathcal{X}^{\prime}\right) \mathcal{J}_{0}-s_{12} c_{13} s_{13}\left(c_{12} s_{13} c_{23} \operatorname{Im} X_{1}\right. \\
& \left.-s_{12} s_{23} \operatorname{Im} X_{2}\right), \\
\mathcal{J}_{e \mu}^{31} \simeq & \left(1-\mathcal{X}^{\prime}\right) \mathcal{J}_{0}-c_{12} c_{13} s_{13}\left(s_{12} s_{13} c_{23} \operatorname{Im} X_{1}\right. \\
& \left.+c_{12} s_{23} \operatorname{Im} X_{2}\right),
\end{aligned}
$$

and

$$
\begin{aligned}
\mathcal{J}_{\tau e}^{12} \simeq & \left(1-\mathcal{Y}^{\prime}\right) \mathcal{J}_{0}+c_{12} s_{12} c_{13}^{3} s_{23} \operatorname{Im} Y_{1} \\
& -c_{12} s_{12} c_{13}^{2} s_{13}\left(c_{23}^{2} \operatorname{Im} Z_{2}+s_{23}^{2} \operatorname{Im} Z_{3}\right), \\
\mathcal{J}_{\tau e}^{23} \simeq & \left(1-\mathcal{Y}^{\prime}\right) \mathcal{J}_{0}-s_{12} c_{13} s_{13}\left(c_{12} s_{13} s_{23} \operatorname{Im} Y_{1}+s_{12} c_{23} \operatorname{Im} Y_{2}\right) \\
& -c_{12} s_{12} c_{13}^{2} s_{13}\left(c_{23}^{2} \operatorname{Im} Z_{2}+s_{23}^{2} \operatorname{Im} Z_{3}\right), \\
\mathcal{J}_{\tau e}^{31} \simeq & \left(1-\mathcal{Y}^{\prime}\right) \mathcal{J}_{0}-c_{12} c_{13} s_{13}\left(s_{12} s_{13} s_{23} \operatorname{Im} Y_{1}-c_{12} c_{23} \operatorname{Im} Y_{2}\right) \\
& -c_{12} s_{12} c_{13}^{2} s_{13}\left(c_{23}^{2} \operatorname{Im} Z_{2}+s_{23}^{2} \operatorname{Im} Z_{3}\right),
\end{aligned}
$$

as well as

$$
\begin{aligned}
\mathcal{J}_{\mu \tau}^{12} \simeq & \left(1-\mathcal{Z}^{\prime}\right) \mathcal{J}_{0}+c_{12} s_{12} c_{13}\left[c_{23}\left(s_{23}^{2}-s_{13}^{2}\right) \operatorname{Im} X_{1}\right. \\
& \left.+s_{23}\left(c_{23}^{2}-s_{13}^{2}\right) \operatorname{Im} Y_{1}\right] \\
& -\left(c_{12}^{2}-s_{12}^{2}\right) c_{13} s_{13} c_{23} s_{23}\left(c_{23} \operatorname{Im} X_{2}-s_{23} \operatorname{Im} Y_{2}\right) \\
& +c_{12} s_{12} c_{13} s_{13}^{2} c_{23} s_{23}\left(s_{23} \operatorname{Im} X_{3}+c_{23} \operatorname{Im} Y_{3}\right) \\
& -\left(c_{12}^{2}-s_{12}^{2}\right) s_{13}^{2} c_{23} s_{23} \operatorname{Im} Z_{1}-c_{12} s_{12} s_{13} c_{23}^{2} \operatorname{Im} Z_{2} \\
& +c_{12} s_{12} s_{13}^{3} s_{23}^{2} \operatorname{Im} Z_{3}, \\
\mathcal{J}_{\mu \tau}^{23} \simeq & \left(1-\mathcal{Z}^{\prime}\right) \mathcal{J}_{0}+c_{12} c_{13}\left[s_{12} c_{23}\left(s_{23}^{2}-s_{13}^{2}\right) \operatorname{Im} X_{1}\right. \\
& \left.+s_{12} s_{23}\left(c_{23}^{2}-s_{13}^{2}\right) \operatorname{Im} Y_{1}+c_{12} c_{13} s_{23} c_{23} \operatorname{Im} Z_{1}\right] \\
& -\left(c_{12}^{2}-s_{12}^{2}\right) c_{13} s_{13} c_{23} s_{23}\left(c_{23} \operatorname{Im} X_{2}-s_{23} \operatorname{Im} Y_{2}\right) \\
& +c_{12} s_{12} c_{13} s_{13}^{2} c_{23} s_{23}\left(s_{23} \operatorname{Im} X_{3}+c_{23} \operatorname{Im} Y_{3}\right) \\
& -c_{12} s_{12} c_{13}^{2} s_{13} s_{23}^{2} \operatorname{Im} Z_{3}, \\
\mathcal{J}_{\mu \tau}^{31} \simeq & \left(1-\mathcal{Z}^{\prime}\right) \mathcal{J}_{0}+s_{12} c_{13}\left[c_{12} c_{23}\left(s_{23}^{2}-s_{13}^{2}\right) \operatorname{Im} X_{1}\right.
\end{aligned}
$$

$$
\begin{aligned}
& \left.+c_{12} s_{23}\left(c_{23}^{2}-s_{13}^{2}\right) \operatorname{Im} Y_{1}-s_{12} c_{13} s_{23} c_{23} \operatorname{Im} Z_{1}\right] \\
& -\left(c_{12}^{2}-s_{12}^{2}\right) c_{13} s_{13} c_{23} s_{23}\left(c_{23} \operatorname{Im} X_{2}-s_{23} \operatorname{Im} Y_{2}\right) \\
& +c_{12} s_{12} c_{13} s_{13}^{2} c_{23} s_{23}\left(s_{23} \operatorname{Im} X_{3}+c_{23} \operatorname{Im} Y_{3}\right) \\
& -c_{12} s_{12} c_{13}^{2} s_{13} s_{23}^{2} \operatorname{Im} Z_{3},
\end{aligned}
$$

where $\mathcal{J}_{0}=c_{12} s_{12} c_{13}^{2} s_{13} c_{23} s_{23} \sin \delta$ with $\delta \equiv \delta_{13}-\delta_{12}-\delta_{23}$, and

$$
\begin{aligned}
X_{1} & =\mathcal{X} e^{-\mathrm{i} \delta_{12}}, \quad X_{2}=\mathcal{X} e^{-\mathrm{i}\left(\delta_{13}-\delta_{23}\right)}, \\
X_{3} & =\mathcal{X} e^{\mathrm{i}\left(\delta_{12}-2 \delta_{13}+2 \delta_{23}\right)}, \\
Y_{1} & =\mathcal{Y} e^{-\mathrm{i}\left(\delta_{12}+\delta_{23}\right)}, \quad Y_{2}=\mathcal{Y} e^{-\mathrm{i} \delta_{13}}, \\
Y_{3} & =\mathcal{Y} e^{\mathrm{i}\left(\delta_{12}-2 \delta_{13}+\delta_{23}\right)}, \\
Z_{1} & =\mathcal{Z} e^{-\mathrm{i} \delta_{23}}, \quad Z_{2}=\mathcal{Z} e^{\mathrm{i}\left(\delta_{12}-\delta_{13}\right)}, \\
Z_{3} & =\mathcal{Z} e^{-\mathrm{i}\left(\delta_{12}-\delta_{13}+2 \delta_{23}\right)},
\end{aligned}
$$

together with

$$
\begin{aligned}
\mathcal{X} & \equiv \hat{s}_{14} \hat{s}_{24}^{*}+\hat{s}_{15} \hat{s}_{25}^{*}+\hat{s}_{16} \hat{s}_{26}^{*}, \\
\mathcal{X}^{\prime} & \equiv s_{14}^{2}+s_{15}^{2}+s_{16}^{2}+s_{24}^{2}+s_{25}^{2}+s_{26}^{2}, \\
\mathcal{Y} & \equiv \hat{s}_{14} \hat{s}_{34}^{*}+\hat{s}_{15} \hat{s}_{35}^{*}+\hat{s}_{16} \hat{s}_{36}^{*}, \\
\mathcal{Y}^{\prime} & \equiv s_{14}^{2}+s_{15}^{2}+s_{16}^{2}+s_{34}^{2}+s_{35}^{2}+s_{36}^{2}, \\
\mathcal{Z} & \equiv \hat{s}_{24} \hat{s}_{34}^{*}+\hat{s}_{25} \hat{s}_{35}^{*}+\hat{s}_{26} \hat{s}_{36}^{*}, \\
\mathcal{Z}^{\prime} & \equiv s_{24}^{2}+s_{25}^{2}+s_{26}^{2}+s_{34}^{2}+s_{35}^{2}+s_{36}^{2} .
\end{aligned}
$$

If the smallness of $\theta_{13}$ is considered and the terms of $\mathcal{O}\left(s_{13}\left|\mathcal{X}^{(\prime)}\right|\right), \mathcal{O}\left(s_{13}\left|\mathcal{Y}^{(\prime)}\right|\right)$ and $\mathcal{O}\left(s_{13}\left|\mathcal{Z}^{(\prime)}\right|\right)$ together with those higher-order terms are omitted in Eqs. (C6)-(C8), then the results in Ref. [28] can be reproduced and one will be left with $\mathcal{J}_{e \mu}^{23} \simeq \mathcal{J}_{e \mu}^{31} \simeq \mathcal{J}_{\tau e}^{23} \simeq \mathcal{J}_{\tau e}^{31} \simeq \mathcal{J}_{0}$.

\section{References}

1. P.A. Zyla et al. [Particle Data Group], PTEP 2020(8), 083C01 (2020)

2. Zz. Xing, Phys. Rept. 854, 1 (2020). arXiv:1909.09610 [hep-ph]

3. B. Pontecorvo, Sov. Phys. JETP 6, 429 (1957)

4. B. Pontecorvo, Zh Eksp, Teor. Fiz. 33, 549 (1957)

5. Z. Maki, M. Nakagawa, S. Sakata, Prog. Theor. Phys. 28, 870 (1962)

6. B. Pontecorvo, Sov. Phys. JETP 26, 984 (1968)

7. B. Pontecorvo, Zh Eksp, Teor. Fiz. 53, 1717 (1967)

8. D. Adey et al. [Daya Bay Collaboration], Phys. Rev. Lett. 121, 241805 (2018). arXiv:1809.02261 [hep-ex] 
9. N. Cabibbo, Phys. Rev. Lett. 10, 531 (1963)

10. M. Kobayashi, T. Maskawa, Prog. Theor. Phys. 49, 652 (1973)

11. K. Abe et al. [T2K Collaboration], Nature 580, 339 (2020). arXiv:1910.03887 [hep-ex]

12. H. Fritzsch, Zz. Xing, Prog. Part. Nucl. Phys. 45, 1 (2000). arXiv:hep-ph/9912358 [hep-ph]

13. J. Aguilar-Saavedra, G. Branco, Phys. Rev. D 62, 096009 (2000). arXiv:hep-ph/0007025 [hep-ph]

14. Zz Xing, H. Zhang, Phys. Lett. B 618, 131 (2005). arXiv:hep-ph/0503118 [hep-ph]

15. S. Luo, Phys. Rev. D 85, 013006 (2012). arXiv: 1109.4260 [hep-ph]

16. Zz Xing, Jy Zhu, Nucl. Phys. B 908, 302-317 (2016). arXiv:1511.00450 [hep-ph]

17. I. Esteban, M.C. Gonzalez-Garcia, M. Maltoni, I. Martinez-Soler, T. Schwetz, JHEP 01, 087 (2017). arXiv:1611.01514 [hep-ph]

18. J.Y. Zhu, Phys. Rev. D 99, 033003 (2019). arXiv:1810.04426 [hep$\mathrm{ph}]$

19. Z.Z. Xing, D. Zhang, Phys. Lett. B 803, 135302 (2020). arXiv:1911.03292 [hep-ph]

20. S.A.R. Ellis, K.J. Kelly, S.W. Li, (2020). arXiv:2004.13719 [hep$\mathrm{ph}]$

21. S.A.R. Ellis, K.J. Kelly, S.W. Li, (2020). arXiv:2008.01088 [hep$\mathrm{ph}]$

22. P. Minkowski, Phys. Lett. 67B, 421 (1977)

23. T. Yanagida, Conf. Proc. C 7902131, 95 (1979)

24. M. Gell-Mann, P. Ramond, R. Slansky, Conf. Proc. C 790927, 315 (1979). arXiv:1306.4669 [hep-th]

25. S.L. Glashow, N.A.T.O. Sci, Ser. B 61, 687 (1980)

26. R.N. Mohapatra, G. Senjanovic, Phys. Rev. Lett. 44, 912 (1980)

27. Zz Xing, Phys. Lett. B 660, 515 (2008). arXiv:0709.2220 [hep-ph]

28. Zz Xing, Phys. Rev. D 85, 013008 (2012). arXiv:1110.0083 [hep$\mathrm{ph}]$

29. M. Lindner, M. Platscher, F.S. Queiroz, Phys. Rept. 731, 1-82 (2018). arXiv:1610.06587 [hep-ph]

30. A. Ilakovac, A. Pilaftsis, Nucl. Phys. B 437, 491 (1995). arXiv:hep-ph/9403398 [hep-ph]

31. R. Alonso, M. Dhen, M.B. Gavela, T. Hambye, JHEP 01, 118 (2013). arXiv:1209.2679 [hep-ph]

32. S.T. Petcov, Sov. J. Nucl. Phys. 25, 340 (1977) (JINR-E2-10176)

33. S.M. Bilenky, S.T. Petcov, B. Pontecorvo, Phys. Lett. B 67, 309 (1977)

34. T.P. Cheng, L.F. Li, Phys. Rev. Lett. 38, 381 (1977)

35. W.J. Marciano, A.I. Sanda, Phys. Lett. B 67, 303-305 (1977)
36. B.W. Lee, S. Pakvasa, R.E. Shrock, H. Sugawara, Phys. Rev. Lett. 38, 937 (1977)

37. B.W. Lee, R.E. Shrock, Phys. Rev. D 16, 1444 (1977)

38. S. Antusch, C. Biggio, E. Fernandez-Martinez, M.B. Gavela, J. Lopez-Pavon, JHEP 10, 084 (2006). arXiv:hep-ph/0607020 [hep$\mathrm{ph}]$

39. S. Antusch, O. Fischer, JHEP 10, 094 (2014). arXiv:1407.6607 [hep-ph]

40. L. Calibbi, G. Signorelli, Riv. Nuovo Cim. 41(2), 71-174 (2018). arXiv:1709.00294 [hep-ph]

41. C. Jarlskog, Phys. Rev. Lett. 55, 1039 (1985)

42. T.P. Cheng, L.F. Li, Phys. Rev. Lett. 45, 1908 (1980)

43. T. Inami, C.S. Lim, Prog. Theor. Phys. 65, 297 (1981)

44. C.S. Lim, T. Inami, Prog. Theor. Phys. 67, 1569 (1982)

45. P. Langacker, D. London, Phys. Rev. D 38, 907 (1988)

46. E. Fernandez-Martinez, J. Hernandez-Garcia, J. Lopez-Pavon, JHEP 08, 033 (2016). arXiv:1605.08774 [hep-ph]

47. S. Goswami, T. Ota, Phys. Rev. D 78, 033012 (2008). arXiv:0802.1434 [hep-ph]

48. M. Drewes, Int. J. Mod. Phys. E 22, 1330019 (2013). arXiv:1303.6912 [hep-ph]

49. J. Hisano, T. Moroi, K. Tobe, M. Yamaguchi, Phys. Rev. D 53, 2442-2459 (1996). arXiv:hep-ph/9510309 [hep-ph]

50. J. Hisano, T. Moroi, K. Tobe, M. Yamaguchi, T. Yanagida, Phys. Lett. B 357, 579-587 (1995). arXiv:hep-ph/9501407 [hep-ph]

51. Z.z. Xing, Z.h. Zhao, (2020). arXiv:2008.12090 [hep-ph]

52. A.J. Buras, P.H. Weisz, Nucl. Phys. B 333, 66-99 (1990)

53. G. 't Hooft, M.J.G. Veltman, Nucl. Phys. B 44, 189-213 (1972)

54. P. Breitenlohner, D. Maison, Commun. Math. Phys. 52, 11-38 (1977)

55. P. Breitenlohner, D. Maison, Commun. Math. Phys. 52, 39 (1977)

56. P. Breitenlohner, D. Maison, Commun. Math. Phys. 52, 55 (1977)

57. G. 't Hooft, M.J.G. Veltman, Nucl. Phys. B 153, 365-401 (1979)

58. G. Passarino, M.J.G. Veltman, Nucl. Phys. B 160, 151-207 (1979)

59. A. Denner, Fortsch. Phys. 41, 307-420 (1993). arXiv:0709.1075 [hep-ph]

60. H.H. Patel, Comput. Phys. Commun. 197, 276-290 (2015). arXiv:1503.01469 [hep-ph]

61. H.H. Patel, Comput. Phys. Commun. 218, 66-70 (2017). arXiv:1612.00009 [hep-ph] 